OPEN ACCESS

Edited by:

Tetsuya Tanaka,

Kagoshima University, Japan

Reviewed by:

Kunlaya Somboonwiwat,

Chulalongkorn University, Thailand

Chaozheng Li,

Sun Yat-sen University, China

*Correspondence:

Yeon Soo Han

hanys@jnu.ac.kr

†These authors have contributed equally to this work

Specialty section: This article was submitted to Invertebrate Physiology, a section of the journal

Frontiers in Physiology

Received: 15 August 2021 Accepted: 28 October 2021

Published: 18 November 2021

Citation:

Bae YM, Jo YH, Patnaik BB,

Kim BB, Park KB, Edosa TT, Keshavarz M, Kojour MAM, Lee YS and Han YS (2021) Tenebrio molitor

Spätzle $1 \mathrm{~b}$ is Required to Confer

Antibacterial Defense Against

Gram-Negative Bacteria by

Regulation of Antimicrobial Peptides.

Front. Physiol. 12:758859.

doi: 10.3389/fphys.2021.758859

\section{Tenebrio molitor Spätzle 1b Is Required to Confer Antibacterial Defense Against Gram-Negative Bacteria by Regulation of Antimicrobial Peptides}

Young Min Bae ${ }^{1 t}$, Yong Hun Jo't, Bharat Bhusan Patnaik², Bo Bae Kim¹, Ki Beom Park', Tariku Tesfaye Edosa1,3, Maryam Keshavarz',4, Maryam Ali Mohammadie Kojour1, Yong Seok Lee ${ }^{5}$ and Yeon Soo Han ${ }^{\text {* }}$

1 Department of Applied Biology, Institute of Environmentally-Friendly Agriculture (IEFA), College of Agriculture and Life Sciences, Chonnam National University, Gwangju, South Korea, ${ }^{2}$ Department of Bio-Science and Bio-Technology, Fakir Mohan University, Balasore, India, ${ }^{3}$ Ethiopian Institute of Agricultural Research, Ambo Agricultural Research Center, Ambo, Ethiopia, ${ }^{4}$ Department of Evolutionary Biology, Institute for Biology-Zoology, Free University of Berlin, Berlin, Germany,

${ }^{5}$ Department of Biology, College of Natural Sciences, Soonchunhyang University, Asan, South Korea

Innate immunity is the ultimate line of defense against invading pathogens in insects. Unlike in the mammalian model, in the insect model, invading pathogens are recognized by extracellular receptors, which activate the Toll signaling pathway through an extracellular serine protease cascade. In the Toll-NF-кB pathway, the extracellular spätzle protein acts as a downstream ligand for Toll receptors in insects. In this study, we identified a novel Spätzle isoform (TmSpz1b) from RNA sequencing database of Tenebrio molitor. TmSpz1b was bioinformatically analyzed, and functionally characterized for the antimicrobial function by RNA interference (RNAi). The $702 \mathrm{bp}$ open reading frame of TmSpz1b encoded a putative protein of 233 amino acid residues. A conserved cystine-knot domain with seven cysteine residues in TmSpz1b was involved in three disulfide bridges and the formation of a spätzle dimer. TmSpz1b was mostly expressed in the hemocytes of T. molitor late instar larvae. The mRNA expression of TmSpz1b was highly induced in the hemocytes after Escherichia coli, Staphylococcus aureus, and Candida albicans stimulation of T. molitor larvae. TmSpz1b silenced larvae were significantly more susceptible to E. coli infection. In addition, RNAi-based functional assay characterized TmSpz1b to be involved in the positive regulation of antimicrobial peptide genes in hemocytes and fat bodies. Further, the TmDorX2 transcripts were downregulated in TmSpz1b silenced individuals upon E. coli challenge suggesting the relationship to Toll signaling pathway. These results indicate that TmSpz1b is involved in the T. molitor innate immunity, causes the sequestration of Gram-negative bacteria by the regulatory action of antimicrobial peptides, and enhances the survival of $T$. molitor larvae.

Keywords: T. molitor, spätzle, innate immunity, antimicrobial peptides, RNA interference 


\section{INTRODUCTION}

Innate immune responses, such as antimicrobial peptide (AMP) production, coagulation, prophenoloxidase cascade, phagocytosis, melanization, nodule formation, and encapsulation processes, are the major defense systems against invading pathogens in invertebrates. AMP production is one of the most important innate immune responses. The Toll and immune deficiency (IMD) signaling pathways are the major immune responses that regulate the production of AMPs in Drosophila (De Gregorio et al., 2002).

The Toll receptor was first identified in Drosophila melanogaster and was reported to be essential for establishment of the dorsal-ventral patterning in the Drosophila embryo. The precursor form of the Toll receptor reportedly converts to an active Toll receptor in a position-dependent manner, relative to the dorsal-ventral axis (Anderson et al., 1985). Since 1995, various research groups have studied the effects of the Toll signaling pathway on innate immune responses against various pathogens. In the Drosophila model, the dorsal gene, a homolog of a rel-related gene acting as a nuclear factor-kappa B (NF-кB), promotes expression of an antifungal peptide, diptericin, through a Toll signaling pathway, defined by Toll or cactus mutant screening (Lemaitre et al., 1995). In addition, the dorso-ventral regulatory gene cassette (spätzleToll-cactus) is involved in the antifungal immune response by regulating the expression of the antifungal peptide gene drosomycin (Lemaitre et al., 1996). Further, it has been suggested that Gram-positive bacteria recognized by the peptidoglycan recognition protein (PGRP) activate the Drosophila Toll pathway (Michel et al., 2001). Moreover, an active form of the spätzle cytokine directly binds to the multimerized Toll receptors to initiate the intracellular Toll signaling pathway (Weber et al., 2003; Hu et al., 2004). Interestingly, recent studies have suggested that the Toll signaling pathway is also required for antiviral immune response against oral infection (Ferreira et al., 2014). In addition, Drosophila antiviral autophagy against vesicular stomatitis virus (VSV) is triggered by the Toll-7 receptor on the plasma membrane (Nakamoto et al., 2012).

The functional role of Toll-like receptors (TLRs) has been well characterized in mammals. In humans, TLRs, which are the homologs of Drosophila Toll receptor, were identified as type I transmembrane proteins that possess an extracellular leucine-rich repeat (LRR) domain that recognizes pathogen associated molecular patterns (PAMPs), and an intracellular Tollinterleukin-1 receptor (TIR) domain that activates downstream signaling (Medzhitov, 2001; Godfroy et al., 2012). TLRs have been classified into two subgroups based on cellular location and PAMP recognition. TLR1, TLR2, TLR4, TLR5, TLR6, and TLR10 reside on the cell membrane and recognize bacterial cell walls. TLR3, TLR7, TLR8, and TLR9 are expressed in intracellular compartments, like endosomes and target bacterial and viral nucleic acids (Kawai and Akira, 2010). The functions of TLR in innate immune signaling have been fully characterized, scrutinized in mammalian models (Yu et al., 2016; Li et al., 2018) and have been summarized (Nie et al., 2018).
The invertebrate Toll pathway includes an extracellular serine protease cascade. In Drosophila, the extracellular ligand for Toll pathway, spätzle, is activated during development by two different enzymes, including Easter (Chasan and Anderson, 1989) and the spätzle processing enzyme (SPE), which are required for innate immunity (Jang et al., 2006). Mature spätzle is important in dorsal-ventral polarity (Schneider et al., 1994; Morisato, 2001) and is required for antifungal immune response in Drosophila (Lemaitre et al., 1996). A recent study showed that the SPE can be activated by injection of Micrococcus luteus and Bacillus subtilis (Yamamoto-Hino and Goto, 2016). Furthermore, the spätzle protein secreted from hemocytes regulates the production of AMPs from fat bodies by septic injury (Shia et al., 2009). The spätzle protein is activated by an extracellular serine protease cascade, and the dimeric active form of spätzle (C106) directly binds to the Toll receptor (Weber et al., 2003; Arnot et al., 2010). Various studies have characterized the innate immune functions of spätzle in other insects. In Aedes aegypti, spätzle1C activates the Toll5A receptor to mediate an antifungal immune response against the entomopathogenic fungus Beauveria bassiana (Shin et al., 2006). In Bombyx mori, the active form of recombinant spätzle1 $(B m S p z 1)$ protein regulates several AMPs, such as attacin, cecropin, gloverin, moricin, and lebocin, unlike the inactive form of recombinant BmSpz1 (Wang et al., 2007). Cleavage of the Spätzle-C108 dimer by extracellular proteolytic cascades activates the Toll pathway in response to a wide variety of microbes. This results in lysozyme stimulation and the production of several AMPs, including attacin-1, cecropin-6, and moricin, in Manduca sexta (An et al., 2010). In another lepidopteran insect, Antheraea pernyi, the induction patterns of Toll pathway-related genes, including those for Gram-negative bacteria binding protein (GNBP), spätzle1, Toll, MyD88, Cactus, and dorsalA, were analyzed after microbial challenges. The Toll pathway-related genes were significantly induced by the injection of fungi (Nosema pernyi) and Grampositive bacteria (Enterococcus pernyi), but not by Gram-negative bacteria (Escherichia coli) (Sun et al., 2016). In the mealworm beetle T. molitor, TmSpz4 and TmSpz6 are required for the regulation of AMP production against E. coli, C. albicans, and $S$. aureus infections, suggesting the involvement of AMP in the increased survival of $T$. molitor threatened with infections (Edosa et al., 2020a,b).

In aquatic invertebrates, such as the marine shrimp Fenneropenaeus chinensis, the spätzle ( $F c S p z)$ gene is induced by the Gram-negative bacterium Vibrio anguillarum and white spot syndrome virus (WSSV) (Shi et al., 2009). Furthermore, injection of the active form of $F c S p z$ can induce several AMP genes in crayfish (Shi et al., 2009). In another marine shrimp, Artemia sinica, a full-length cDNA sequence of the spätzle gene belonging to spätzle-4 family was identified. The gene was highly induced by injection of Gram-positive bacteria, such as Micrococcus lysodeikticus, suggesting an important function in innate immunity (Zheng et al., 2012). In Macrobrachium rosenbergii, microbial susceptibility against the Gram-negative bacterium Aeromonas caviae was significantly increased by silencing of $M r S p z$ in shrimp (Vaniksampanna et al., 2019). The first mollusk spätzle homolog gene was 
identified in the clam Paphia undulate and was shown to be involved in the host defense against both Gramnegative ( $V$. alginolyticus) and Gram-positive bacteria (Listeria monocytogenes) (Yu et al., 2015).

In the beetle model, the serine protease signaling cascade for extracellular Toll signaling pathway has been fully characterized by elegant studies using biochemical and molecular approaches. Lysine-type peptidoglycan (PG) recognition complex initially recognizes pathogenic patterns, followed by a three-step proteolytic cascade that finally cleaves the spätzle protein to activate a PG-dependent Toll signaling pathway (Kim et al., 2008; Ali Mohammadie Kojour et al., 2020). Moreover, the fungal cell wall component $\beta$-1, 3-glucan also activates the T. molitor Toll pathway (Roh et al., 2009). In our recent study on RNA interference (RNAi)-based functional characterization of immune-related genes revealed that TmCactin, a Tenebrio cactus binding protein, was activated by Gram-negative and Gram-positive bacteria, and promiscuously regulated five AMP genes (Jo et al., 2017). Another component of the Toll signaling pathway, TmToll-7, was interestingly activated by the Gramnegative bacterium, E. coli, and positively regulated seven AMP genes (Park et al., 2019). In addition, our recent bioinformatics analysis identified nine spätzle isoforms from the $T$. molitor model (TmSpz-like, -1b, -3, -4, -5, -6, -7, -7a, -7b). Apart from Spätzle4 and Spätzle6 that has been discussed in the context of humoral immunity in T. molitor the functions of other spätzle isoforms are still elusive. TmSpz4 regulates AMP production against $E$. coli and C. albicans infection through the activation of Toll pathway (Edosa et al., 2020a) and TmSpz6 regulates AMP expression and increases survival of T. molitor against E. coli and S. aureus (Edosa et al., 2020b).

In the present study, a novel spätzle isoform, TmSpz1b, was functionally characterized for its function in regulating AMP production against Gram-negative bacteria, but not against Gram-positive bacteria and fungus. The downregulated TmSpz1b transcript resulted in high cumulative mortality of $E$. coli-infected T. molitor larvae. The results suggest that TmSpzlb is involved in T. molitor innate immunity, causing the sequestration of Gram-negative bacteria by the regulatory action of antimicrobial peptides, and enhances survival of T. molitor larvae.

\section{MATERIALS AND METHODS}

\section{Insect Rearing}

Larvae of the yellow mealworm beetle (T. molitor) were reared under continuous dark conditions at $27 \pm 1^{\circ} \mathrm{C}$ and $60 \% \pm 5 \%$ relative humidity (R.H.) in an environmental chamber. The reared larvae were fed an artificial diet consisting of $170 \mathrm{~g}$ wheat flour, $20 \mathrm{~g}$ roasted soy flour, $10 \mathrm{~g}$ protein, $100 \mathrm{~g}$ wheat bran, $0.5 \mathrm{~g}$ sorbic acid, $0.5 \mathrm{~mL}$ propionic acid, and $0.5 \mathrm{~g}$ chloramphenicol in $200 \mathrm{~mL}$ of distilled water (D.W.). Only 10th to 12th instar T. molitor larvae were used in these experiments.

\section{Preparation of Microorganisms}

The Gram-negative bacterium E. coli (strain K12), Gram-positive bacterium S. aureus (strain RN4220), and the fungus C. albicans (strain AUMC 13529) were used for the immune challenge studies. E. coli and S. aureus were cultured overnight in LuriaBertani broth (MB Cell, Seoul, Korea) at $37^{\circ} \mathrm{C}$. C. albicans was cultured overnight at $37^{\circ} \mathrm{C}$ in Sabouraud dextrose broth (MB Cell). The microorganisms were harvested and washed twice in $1 \times$ phosphate-buffered saline (PBS; $8.0 \mathrm{~g} \mathrm{NaCl}, 0.2 \mathrm{~g} \mathrm{KCl}$, $1.42 \mathrm{~g} \mathrm{Na}_{2} \mathrm{HPO}_{4}$, and $0.24 \mathrm{~g} \mathrm{KH}_{2} \mathrm{PO}_{4}$ in $1 \mathrm{l}$ of D.W; $\mathrm{pH}$ 7.0), and centrifuged at 3,500 rpm for $10 \mathrm{~min}$. Each cell pellet was subsequently suspended in PBS, and the concentrations of microorganisms were measured by their optical density at $600 \mathrm{~nm}\left(\mathrm{OD}_{600}\right)$ by spectrophotometry (Eppendorf, Hamburg, Germany). The suspensions were adjusted to $1 \times 10^{6}$ cells/ $\mu \mathrm{l}$ (E. coli and S. aureus) or $5 \times 10^{4}$ cells/ $\mu l$ (C. albicans) for immune challenge studies.

\section{Identification and in silico Analysis of T. molitor Spätzle1b}

To identify the TmSpz1b gene (Accession no. MZ708791), a localtblastn analysis was performed using the amino acid sequence of Tribolium castaneum spätzle X3 (TcSpzX3) (GenBank: XP015840683.1) as a query against the locally curated T. molitor nucleotide database derived from T. molitor RNA sequencing. The deduced amino acid sequence of TmSpzlb was analyzed using the blastx and blastp algorithms (Mount, 2007) at NCBI. The full-length target open reading frame (ORF) region were amplified by AccuPower Pfu Pre-Mix (Bioneer, Daejeon, South Korea) on a MyGenie 96 thermal block (Bioneer) using gene-specific primers designed using Primer 3.0 software $^{1}$ (Table 1). The PCR purified products were cloned into the T-blunt vector cloning system (Solgent Company, Daejeon, South Korea), transformed into E. coli DH5 $\alpha$ cells, and sequenced using M13 primers. After sequencing the full-length ORF sequence was validated.

\section{Domain and Phylogenetic Analyses}

The domain architecture of the protein sequences were retrieved using the InterProScan 5.0 (Jones et al., 2014) and blastp (Mount, 2007) programs. The signal peptide was predicted using the SignalP 5.0 server $^{2}$. The expasy server tools at Swiss Institute of Bioinformatics ${ }^{3}$, including 'Compute $\mathrm{Pi} / \mathrm{MW}$ ' and 'ProtParam,' were used to identify the physico-chemical properties of the putative protein. The NetPhos 3.1 prediction tool at https:// services.healthtech.dtu.dk/ was used to predict serine, threonine, or tyrosine phosphorylation sites in the TmSpz1b protein.

Multiple sequence alignment profile was used to estimate the genetic relatedness of TmSpzlb among Spätzle genes representing different insect orders obtained from GenBank using ClustalX v 2.1 (Larkin et al., 2007) software. Only the amino acid sequence of specific cystine-knot cytokine domain of TmSpz1b was used. The .pim output files from ClustalX v. 2.1 were used to analyze the percentage identity among the insect spätzle sequences from orthologous species. A phylogenetic tree was constructed based on the amino acid sequences of the

\footnotetext{
${ }^{1}$ http://bioinfo.ut.ee/primer3-0.4.0/

${ }^{2}$ http://www.cbs.dtu.dk/services/SignalP/

${ }^{3}$ www.expasy.org
} 
TABLE 1 | Primers used in the study.

\begin{tabular}{|c|c|}
\hline Name & Primer sequences \\
\hline $\begin{array}{l}\text { TmSpz1b_cloning_Fw } \\
\text { TmSpz1b_cloning_Rv }\end{array}$ & $\begin{array}{l}\text { 5'-TACAGGTCAACCCCAAGACC-3' } \\
\text { 5'-CGACGGCACTITAAACGAAT-3' }\end{array}$ \\
\hline $\begin{array}{l}\text { TmSpz1b_qPCR_Fw } \\
\text { TmSpz1b_qPCR_Rv }\end{array}$ & $\begin{array}{l}\text { 5'-GGACGCTTCCCATTAGTGCT-3' } \\
\text { 5'TCTAAGTGTGAATGCGCCGT-3' }\end{array}$ \\
\hline $\begin{array}{l}\text { TmSpz1b_T7_Fw } \\
\text { TmSpz1b_T7_Rv }\end{array}$ & $\begin{array}{l}5^{\prime} \text {-TAATACGACTCACTATAGGGT } \\
\text { GCTGGCTACCCAAAAGAACA-3' } \\
5^{\prime} \text {-TAATACGACTCACTATAGGGT } \\
\text { CGACGGCACTTAAACGAAT-3' }\end{array}$ \\
\hline $\begin{array}{l}\text { EGFP_T7_Fw } \\
\text { EGFP_T7_Rv }\end{array}$ & 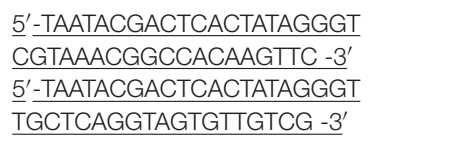 \\
\hline $\begin{array}{l}\text { TmTenecin-1_qPCR_Fw } \\
\text { TmTenecin-1_qPCR_Rv }\end{array}$ & $\begin{array}{l}\text { 5'-CAGCTGAAGAAATCGAACAAGG-3' } \\
\text { 5'-CAGACCCTCTTCCGTACAGT-3' }\end{array}$ \\
\hline $\begin{array}{l}\text { TmTenecin-2_qPCR_Fw } \\
\text { TmTenecin-2_qPCR_Rv }\end{array}$ & $\begin{array}{l}\text { 5'-CAGCAAAACGGAGGATGGTC-3' } \\
\text { 5'-CGTTGAAATCGTGATCTTGTCC-3' }\end{array}$ \\
\hline $\begin{array}{l}\text { TmTenecin-3_qPCR_Fw } \\
\text { TmTenecin-3_qPCR_Rv }\end{array}$ & $\begin{array}{l}\text { 5'-GATTTGCTTGATTCTGGTGGTC-3' } \\
\text { 5'-CTGATGGCCTCCTAAATGTCC-3' }\end{array}$ \\
\hline $\begin{array}{l}\text { TmTenecin-4_qPCR_Fw } \\
\text { TmTenecin-4_qPCR_Rv }\end{array}$ & $\begin{array}{l}\text { 5'-GGACATTGAAGATCCAGGAAAG-3' } \\
\text { 5'-CGGTGTTCCTTATGTAGAGCTG-3' }\end{array}$ \\
\hline $\begin{array}{l}\text { TmDefensin_qPCR_Fw } \\
\text { TmDefensin_qPCR_Rv }\end{array}$ & $\begin{array}{l}\text { 5'-AAATCGAACAAGGCCAACAC-3' } \\
\text { 5'-GCAAATGCAGACCCTCTTC-3' }\end{array}$ \\
\hline $\begin{array}{l}\text { TmDefensin-like_qPCR_Fw } \\
\text { TmDefensin-like_qPCR_Rv }\end{array}$ & $\begin{array}{l}\text { 5'-GGGATGCCTCATGAAGATGTAG-3' } \\
\text { 5'-CCAATGCAAACACATTCGTC-3' }\end{array}$ \\
\hline $\begin{array}{l}\text { TmColeoptericin-A_qPCR_Fw } \\
\text { TmColeoptericin-A_qPCR_Rv }\end{array}$ & $\begin{array}{l}\text { 5'-GGACAGAATGGTGGATGGTC-3' } \\
\text { 5'-CTCCAACATTCCAGGTAGGC-3' }\end{array}$ \\
\hline $\begin{array}{l}\text { TmColeoptericin-B_qPCR_FW } \\
\text { TmColeoptericin-B_qPCR_Rv }\end{array}$ & $\begin{array}{l}\text { 5'-CAGCTGTTGCCCACAAAGTG-3' } \\
\text { 5'-CTCAACGTTGGTCCTGGTGT-3' }\end{array}$ \\
\hline $\begin{array}{l}\text { TmAttacin-1a_qPCR_Fw } \\
\text { TmAttacin-1a_qPCR_Rv }\end{array}$ & $\begin{array}{l}\text { 5'-AAAGTGGTCCCCACCGATTC-3' } \\
\text { 5'-GCGCTGAATGTITCGGCTT-3' }\end{array}$ \\
\hline $\begin{array}{l}\text { TmAttacin-1b_qPCR_Fw } \\
\text { TmAttacin-1b_qPCR_Rv }\end{array}$ & $\begin{array}{l}\text { 5'-GAGCTGTGAATGCAGGACAA-3' } \\
\text { 5'-CCCTCTGATGAAACCTCCAA-3' }\end{array}$ \\
\hline $\begin{array}{l}\text { TmAttacin-2_qPCR_Fw } \\
\text { TmAttacin-2_qPCR_Rv }\end{array}$ & $\begin{array}{l}\text { 5'-AACTGGGATATTCGCACGTC-3' } \\
\text { 5'-CCCTCCGAAATGTCTGTTGT-3' }\end{array}$ \\
\hline $\begin{array}{l}\text { TmCecropin-2_qPCR_Fw } \\
\text { TmCecropin-2_qPCR_Rv }\end{array}$ & $\begin{array}{l}\text { 5'-TACTAGCAGCGCCAAAACCT-3' } \\
\text { 5'-CTGGAACATTAGGCGGAGAA-3' }\end{array}$ \\
\hline $\begin{array}{l}\text { TmTLP1_qPCR_Fw } \\
\text { TmTLP1_qPCR_Rv }\end{array}$ & $\begin{array}{l}\text { 5'-CTCAAAGGACACGCAGGACT-3' } \\
\text { 5'-ACTTTGAGCTTCTCGGGACA-3' }\end{array}$ \\
\hline $\begin{array}{l}\text { TmTLP2_qPCR_FW } \\
\text { TmTLP2_qPCR_Rv }\end{array}$ & $\begin{array}{l}\text { 5'-CCGTCTGGCTAGGAGTTCTG-3' } \\
\text { 5'-ACTCCTCCAGCTCCGTTACA-3' }\end{array}$ \\
\hline $\begin{array}{l}\text { TmDorX1_qPCR_Fw } \\
\text { TmDorX1_qPCR_Rv }\end{array}$ & $\begin{array}{l}\text { 5'-AGCGTTGAGGTTCGGTATG-3' } \\
\text { 5'-TCTITGGTGACGCAAGACAC-3' }\end{array}$ \\
\hline $\begin{array}{l}\text { TmDorX2_qPCR_Fw } \\
\text { TmDorX2_qPCR_Rv }\end{array}$ & $\begin{array}{l}\text { 5'-ACACCCCCGAAATCACAAAC-3' } \\
5^{\prime} \text {-TTCAGAGCGCCAGGTITG-3' }\end{array}$ \\
\hline $\begin{array}{l}\text { TmRelish_qPCR_Fw } \\
\text { TmRelish_qPCR_Rv }\end{array}$ & $\begin{array}{l}\text { 5'-AGCGTCAAGTTGGAGCAGAT-3' } \\
\text { 5'-GTCCGGACCTCAAGTGT-3' }\end{array}$ \\
\hline $\begin{array}{l}T m L 27 \text { a_qPCR_Fw } \\
T m L 27 \text { a_qPCR_Rv }\end{array}$ & $\begin{array}{l}\text { 5'-TCATCCTGAAGGCAAAGCTCCAGT-3' } \\
\text { 5'-AGGTTGGTTAGGCAGGCACCTTA-3' }\end{array}$ \\
\hline
\end{tabular}

Underline indicates $T 7$ promotor sequences.

TmSpz1b gene by using the neighbor-joining (NJ) method in the MEGA v. 7.0 software program (Kumar et al., 2016). The bootstrap consensus tree was inferred from 1000 replicates, and the evolutionary distances were computed using the Poisson correction method. The amino acid sequence of Penaeus vannamei spätzle ( $P v \mathrm{Spz}$; ROT72693.1) was used as an outgroup for this analysis.

\section{Developmental, Tissue-Specific Expression, and Induction of TmSpz1b mRNA in Response to Pathogenic Challenges}

To investigate the developmental expression patterns of TmSpz1b mRNA, whole body samples $(n=20$ for each stage) were collected from young larvae (YL; 10th-12th instar larvae), late instar larvae (LL; 19th-20th instar larvae), pre-pupae (PP), 1-7-day old pupae (P1-P7), and 1-5-day old adults (A1-A5). To investigate the tissue-specific expression profiles, integument (IT), fat body (FB), hemocytes (HC), gut (GT), and Malpighian tubules (MT) were dissected from both late instar larvae and 5-day old adults, and ovary (OV) and testes (TE) were collected only from 5-day old adults of T. molitor.

To investigate the expression and induction patterns of TmSpz1b mRNA, prepared microorganisms including E. coli, S. aureus, and C. albicans, were injected into 11th-12th instar larvae $(n=20)$ of T. molitor. PBS injected T. molitor group was used as a mock control. The hemocytes, fat body and gut were dissected at $3,6,9,12$, and $24 \mathrm{~h}$ postinoculation of microorganisms. The samples were stored at $-80^{\circ} \mathrm{C}$ for further use.

Total RNA was extracted by a Clear-S ${ }^{\mathrm{TM}}$ Total RNA Extraction Kit (Invirustech Co., Gwangju, South Korea). To synthesize cDNA, total RNA $(2 \mu \mathrm{g})$ was used as the template with an Oligo $(\mathrm{dT})_{12-18}$ primer at $72^{\circ} \mathrm{C}$ for $5 \mathrm{~min}, 42^{\circ} \mathrm{C}$ for $1 \mathrm{~h}$, and $94^{\circ} \mathrm{C}$ for $5 \mathrm{~min}$ on a MyGenie96 Thermal Block (Bioneer) and using AccuPower ${ }^{\circledR}$ RT PreMix (Bioneer) according to manufacturer's instructions. CDNA was stored at $-20^{\circ} \mathrm{C}$ until further use.

The relative expression level of TmSpz1b mRNA was investigated by performing quantitative real-time polymerase chain reaction (qRT-PCR) using AccuPower ${ }^{\circledR} 2 \mathrm{X}$ Greenstar ${ }^{\mathrm{TM}}$ qPCR Master Mix (Bioneer), with synthesized cDNAs, and $T m S p z 1 b$ gene-specific primers designed using the Primer 3 plus program $^{4}$, as listed in Table 1. The qRT-PCR was programmed at an initial denaturation of $95^{\circ} \mathrm{C}$ for $5 \mathrm{~min}$, followed by 40 cycles of denaturation at $95^{\circ} \mathrm{C}$ for $15 \mathrm{~s}$, and annealing and extension at $60^{\circ} \mathrm{C}$ for $30 \mathrm{~s}$. The qRT-PCR assays were performed on an AriaMx Real-Time PCR System (Agilent Technologies, Santa Clara, CA, United States), and the results were analyzed using AriaMx Real-Time PCR software. T. molitor ribosomal protein L27a (TmL27a) was used as an internal control, and the mRNA expression levels were analyzed by using $2^{-\Delta \Delta \mathrm{Ct}}$ methods (Livak and Schmittgen, 2001). The results represent mean \pm SE of three biological replications.

\section{Synthesis of Double-Stranded RNA}

Double-stranded RNAs (dsRNA) for the TmSpz1b gene were synthesized to perform RNAi experiments. For the synthesis of dsRNA, the TmSpz1b DNA fragment was amplified by PCR using gene-specific primers tailed $\left(5^{\prime}\right.$ end) with a T7 promoter sequence (Table 1). The primers were designed

\footnotetext{
${ }^{4}$ http://primer3plus.com/cgi-bin/dev/primer3plus.cgi
} 
using SnapDragon software ${ }^{5}$ to prevent any cross-silencing effects. PCR products were amplified using AccuPower ${ }^{\circledR}$ Pfu PCR PreMix under the following cycling conditions: an initial denaturation step at $94^{\circ} \mathrm{C}$ for $5 \mathrm{~min}$, followed by 30 cycles of denaturation at $94^{\circ} \mathrm{C}$ for $30 \mathrm{~s}$, annealing at $53^{\circ} \mathrm{C}$ for $40 \mathrm{~s}$, and extension at $72^{\circ} \mathrm{C}$ for $40 \mathrm{~s}$ on a MyGenie96 Thermal Block (Bioneer). The PCR products were purified by the AccuPrep PCR Purification Kit (Bioneer), and dsRNA was synthesized from purified PCR products $(1 \mu \mathrm{g})$ using the EZ ${ }^{\mathrm{TM}}$ T7 High Yield in Vitro Transcription Kit (Enzynomics, Daejeon, South Korea), according to the manufacturer's instructions. The dsRNA product was purified by the Phenol: Chloroform: Isoamyl alcohol mixture (PCI) method, precipitated with $5 \mathrm{M}$ ammonium acetate, and washed with 70 and $90 \%$ ethanol. Subsequently, it was quantified using an Epoch spectrophotometer (BioTek Instruments, Inc., Winooski, VT, United States). The synthesized dsRNA was stored at $-20^{\circ} \mathrm{C}$ until further use.

For the knockdown validation of TmSpz1b mRNA, $1 \mu \mathrm{g} / \mu \mathrm{l}$ of synthesized dsRNA of Enhanced green fluorescent protein (EGFP) and TmSpz1b were injected into T. molitor young-instar larvae (10th-12th instars; $n=20$ ) by using disposable capillary needles mounted on a micro-applicator (Picospiritzer III Micro Dispense System; Parker Hannifin, Hollis, NH, United States). EGFP dsRNA synthesized from pEGFP-C1 plasmid DNA was used as a negative control for RNAi.

\section{Mortality Assay}

To measure the cumulative mortality in TmSpz1b knockdown T. molitor larvae, healthy larvae were injected with $1 \mu \mathrm{g} / \mu \mathrm{l}$ of dsTmSpz1b or dsEGFP. Subsequently, E. coli, S. aureus and C. albicans were injected into TmSpz1b silenced T. molitor larvae. Dead larvae were counted daily for up to 10 days. Ten insect larvae were used for each set of mortality assays, and the experiments were repeated in triplicate. The results were obtained by Kaplan-Meier survival analysis (Goel et al., 2010).

\section{Effect of TmSpz1b RNAi on Antimicrobial Peptide Gene Expression}

To further characterize the immunological function of TmSpz1b gene in humoral innate immune response, the effect of TmSpz1b silencing by RNAi on the expression levels of 14 AMP genes against microbial challenge were investigated. Two days posttreatment of TmSpz1b dsRNA into T. molitor larvae, these larvae were injected with $E$. coli or $S$. aureus $\left(1 \times 10^{6}\right.$ cells/larva), or C. albicans $\left(5 \times 10^{4}\right.$ cells/larva). After $24 \mathrm{~h}$, immune organs that included hemocytes, fat bodies, and the gut were dissected. Total RNA was extracted, and cDNA was synthesized as described above. The dsEGFP-treated T. molitor larvae and PBS were used as the negative and injection controls, respectively.

Expression patterns of 14 AMP genes including TmTenecin1, 2, 3, and 4 (TmTene1, $-2,-3$, and -4) (Kim et al., 1998; Chae et al., 2012; Yang et al., 2017), TmDefensin and TmDefensin-like (TmDef and TmDef-like) (Jang et al., 2020b), TmColeoptericin$A$ and $-B$ (TmColeA and $-B$ ) (Zhu et al., 2014; Jang et al., 2020a), TmAttacin-1a, $-1 b$ and -2 (TmAtt1a, $-1 b$ and -2)

${ }^{5}$ http://www.flyrnai.org/cgi-bin/RNAi_find_primers.pl
(Jo et al., 2018), TmCecropin-2 (TmCec2) (Ali Mohammadie Kojour et al., 2021), and TmThaumatin-like protein-1 and -2 (TmTLP1 and -2) (Noh and Jo, 2016; Kim et al., 2017), were examined by qRT-PCR with the AMP gene-specific primers (Table 1). A relative quantitative PCR was performed as detailed above in an AMP-specific primer.

\section{Statistical Analysis}

The statistical analysis was performed by one-way analysis of variance (ANOVA) and Tukey's multiple range tests were used to estimate the difference between groups $(p<0.05)$.

\section{RESULTS}

\section{Identification and in silico Analysis of TmSpz1b Genes}

The TmSpzlb gene was identified using in silico protocols. A tblastn analysis with the amino acid sequence of T. castaneum spätzle 1b (XP_975083.1) as query against the T. molitor RNA sequencing database was useful to screen TmSpz1b. The obtained $T m S p z 1 b$ nucleotide sequence was confirmed by blastx analysis ${ }^{6}$ against the GenBank nr database. The ORF of TmSpz1b was confirmed by cloning and sequencing. The full-length cDNA of TmSpzlb was 1,650 bp in length, including a 627 bp and 276 bp $5^{\prime}$ - and $3^{\prime}$ - untranslated region (UTR), respectively, excluding the poly-A tail. A polyadenylation signal (5'-AATAAA- $\left.3^{\prime}\right)$ was located $11 \mathrm{bp}$ upstream of the poly-A tail sequence. The $702 \mathrm{bp}$ ORF of TmSpz1b encoded a putative protein of 233 amino acids (Figure 1) with a calculated molecular weight of $26.38 \mathrm{kDa}$ and a pI of 8.33 . The total number of positively charged residues $($ Asp + Glu) in TmSpz1b was 25. The total number of negatively charged residues (Arg + Lys) was 28. TmSpz1b had an extinction coefficient of 12,420 (Cys form Cystines) and 11,920 (Cys are reduced), with an instability index, aliphatic index, and grand average of hydropathicity of 35.29 (indicating a stable protein), 69.74 , and -0.412 , respectively.

To determine the structure of this gene, domain analysis was performed using the blastp and InterProScan 5 programs. The findings indicated that TmSpzlb possesses a C-terminal cystine-knot domain (spätzle superfamily domain; pfam 16077), a signal peptide region (cleavage site between amino acid positions 22 and 23), and a putative cleavage site. In the cystineknot domain, seven conserved cystine residues were located, forming three disulfide bridges, and one cysteine was involved in the dimerization process. A total of 46 phosphorylation sites were predicted on Ser, Thr, or Tyr residues in TmSpzlb. No glycosylation sites were found.

To understand the evolutionary relationship between TmSpzlb and other insect spätzle proteins, multiple alignment and phylogenetic analyses of insect cystine-knot domains (highly conserved domains of spätzle proteins) were performed using the clustalX2 and MEGA X programs. The multiple alignments of cystine-knot domains showed that seven cystine residues mainly involved in structure formation were well conserved in insects,

\footnotetext{
${ }^{6}$ https://blast.ncbi.nlm.nih.gov/Blast.cgi
} 
TGT AAT TGC GGG AAG CTC TTT ATT CCG AAA CTC GTG TCG GAA ATT TAA CCG CAG GAG TAG ATC TCG AGT GTT GCG TGA TGC GCA AGA GGT GCA CTG CGA ATA AAT GGC ATT AAA TGT TTT GTT TTC ACG TAA TTC AAC GAT TTA TCA TTG AAC ACG ACT TTT ATT TTT TCC ATA GTA ATT AAg CCG CAT TAG TGA TTC AGC CTA CCA AGA AGC AAC AAA TGC GAg CAA ATG TAG ATG TTT CTA ATA AAT TAT TTT GTA CCC ACT TCC GCC TAC AAA TTC GCA CAA ACG CAT TTA CAT CCA CCG GAA AAA CTA CAA TAA CGC TTC CGG GTA ATT CTT TAG CGC GAG AGA TCA TCT TGA AAA AAC CAG ATT TTG CGT GAA AGC GAA CCG GAT GAA TTT TCC TAT TTT ACT GAC CAA TGG TGT AGC CCC TAC GTT CAA CTT GGT GTC CTT GTC GAT ACT CAA CAA TTT CAA TGT TGT TGT GTT GAG TAC AGC AGT AGT GTA ATG TTG TGG TCG GTC AGA TGC TGA ACG CCC GCT AAA ATC AGA TGT CCA GCT CCT TCA GAT GCA TTA TTT CGT ATC ATG TCC AAA CGg GAT AAT TGC TAA CTA GTG CTG CGA GCA ATT CTG ATT TTG ATA

ATG GCA ATG AAT GGg ATT GTG AAG ATA ATA GTT GTC TTA TTG TCC TTG ACG CTT CCC ATT 60

$\begin{array}{lllllllllllllllllllll}M & \text { A } & \text { M } & \text { N } & \text { G } & \text { I } & \text { V } & \text { K } & \text { I } & \text { I } & \text { V } & \text { V } & \text { I } & \text { I } & \text { S } & \text { I } & \text { T } & \text { I } & \text { P } & \text { I } & 20\end{array}$

AGT GCT TCG ACA GAC TAT CTC TAC AGG TCA ACC CCA AGA CCA CGA GGg AGg CCA CAC ATG 120

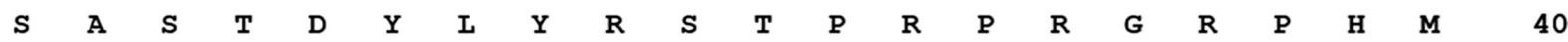

AGT GAT ACA TCG ACC AGA AAT CGA TAT CAC GAG GGC GCA ATT GAA ACA ACG GCG CAT TCA 180

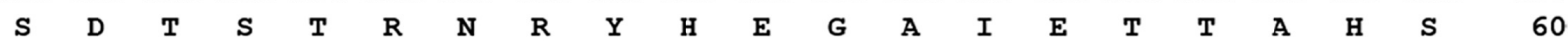

CAC TTA GAT GCA AGA AGT GTT GAC AAA AAA CAA AGG GAG CAC GTA AAC GAC GGT CCA ATA 240

$\begin{array}{lllllllllllllllllllll}\text { H } & \text { L } & \text { D } & \text { A } & \text { R } & \text { S } & \text { V } & \text { D } & \text { K } & \text { K } & \text { Q } & \text { R } & \text { E } & \text { H } & \text { V } & \text { N } & \text { D } & \text { G } & \text { P } & \text { I } & 80\end{array}$

GTT TAC CCA GAG GCC AGC ACA TGC ACC CAT GGC TTG TGC GTC AAC GTC GCT GGC TAC CCA 300

$\begin{array}{lllllllllllllllllllll}\text { V } & \text { Y } & \text { P } & \text { E } & \text { A } & \text { S } & \text { T } & \text { C } & \text { T } & \text { H } & \text { G } & \text { L } & \text { C } & \text { V } & \text { N } & \text { V } & \text { A } & \text { G } & \text { Y } & \text { P } & 100\end{array}$

AAA GAA CAA ATT AAA AAA CTA CTG AGC AGA TCT CAG TTT ATG AAC AGC TAC TTC CAA GCT 360

$\begin{array}{llllllllllllllllllllll}\text { K } & \text { E } & Q & \text { I } & \text { I } & \text { K } & \text { K } & \text { L } & \text { L } & \text { S } & \text { R } & \text { S } & \text { Q } & \text { F } & \text { M } & \text { N } & \text { S } & \text { Y } & \text { F } & \text { Q } & \text { A } & 120\end{array}$

ACC GAA GAG TTC GTC AAT GTA GAT AAT AGG TTT AAT ACG GAT GAg ATG TCG TTG TGT GAA 420

$\begin{array}{llllllllllllllllllllll}\mathbf{T} & \mathrm{E} & \mathrm{E} & \mathrm{F} & \mathrm{V} & \mathrm{N} & \mathrm{V} & \mathrm{D} & \mathrm{N} & \mathrm{R} & \mathrm{F} & \mathrm{N} & \mathrm{T} & \mathrm{D} & \mathrm{E} & \mathrm{M} & \mathrm{S} & \mathrm{L} & \mathrm{C} & \mathrm{E} & 140\end{array}$

ACA AAG GTC CAT ACG ATT TAC CCC GAG AAA GCC AAT AAC ACG ATG GAA ATC GAA AAA GTG 480

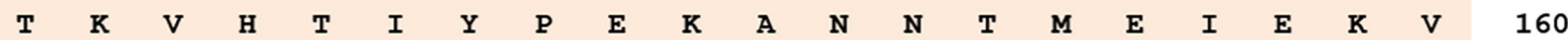

ATT GTC AAC GTG GAA GGA CAC AAA CAA GGG GTG GTT TTC GAG ACT TGC GTG AAC AAC GGG 540

$\begin{array}{llllllllllllllllllllll}\text { I } & \text { V } & \text { N } & \text { V } & \text { E } & \text { G } & \text { H } & \text { K } & \text { Q } & \text { G } & \text { V } & \text { V } & \text { F } & \text { E } & \text { T } & \text { C } & \text { V } & \text { N } & \text { N } & \text { G } & 180\end{array}$

AAA TGT AAA TTT AGC AGC AAT TTT CCG ACT GGA TAC ACA TCT TTC TGT CAA CAA AAA TTC 600

$\begin{array}{llllllllllllllllllllll}\mathbf{K} & \mathbf{C} & \mathrm{K} & \mathbf{F} & \mathbf{S} & \mathbf{S} & \mathbf{N} & \mathbf{F} & \mathbf{P} & \mathbf{T} & \mathbf{G} & \mathbf{Y} & \mathbf{T} & \mathbf{S} & \mathbf{F} & \mathbf{C} & \boldsymbol{Q} & \boldsymbol{Q} & \mathrm{K} & \mathbf{F} & 200\end{array}$

ATC CAC AAg AGg TTG ATG GTC TTG GGA GAT AAC GAT AAg TTt GTG TTC GAT TCG TTT AAA 660

$\begin{array}{llllllllllllllllllllll}\text { I } & \text { H } & \text { K } & \text { R } & \text { L } & \text { M } & \text { V } & \text { L } & \text { G } & \text { D } & \text { N } & \text { D } & \text { K } & \text { F } & \text { V } & \text { F } & \text { D } & \text { S } & \text { F } & \text { K } & 220\end{array}$

GTG CCG TCG TGT TGT GTT TGT ACA GTG ACC AGA AGT GAT TAA

702

$\begin{array}{lllllllllllllllllll}\mathrm{V} & \mathrm{P} & \mathrm{S} & \mathrm{C} & \mathrm{C} & \mathrm{V} & \mathrm{C} & \mathrm{T} & \mathrm{V} & \mathrm{T} & \mathrm{R} & \mathrm{S} & \mathrm{D} & * & & & \end{array}$

ATG GTT ATG TCA CAT TTA TTT CAg ATG TAC AGT GTA TCA GCC TAA TTG GTC GTG TAA TCT TTT GTT TAT TTA TAC AGA TTT TGT TAC TGA GAC ATT CAG TCT TCG ACT TTT GAC TTA CAA CTA TTA ACC TTG TCG TTT ATT GAC TAT TTC TTC TAG GAA TGT GTA AGA TTC AGT TAT TTT ATT GTT GCT TAT AAC AAC ACA CCT ATC ATA TTT GTT CAA AAA TCA ACG CGG AAA ATA AAG TAT AAA ATA ATG AGA GTA ACT GGA ATA AAA CAT GAg AAA TTA AAA AAA AAA AAA AAA AAA AAA

FIGURE 1 | Nucleotide and deduced amino acid sequences of TmSpz1b. TmSpz1b includes an ORF sequence of 702 bp encoding a polypeptide of 233 amino acid sequences. The $5^{\prime}$ - and $3^{\prime}$-UTRs are $627 \mathrm{bp}$ - and $276 \mathrm{bp}$ long, respectively, excluding the poly-A tail. A polyadenylation signal sequence (5'-AATAAA- $\left.3^{\prime}\right)$ is marked with blue text. Nucleotides and amino acids are numbered on the right of the sequences. ${ }^{*}$ Denotes stop codon. Domain analysis of $T m S p z 1 \mathrm{~b}$ revealed a C-terminal cystine-knot domain (orange box), a signal peptide region (cleaving site between amino acids 22 and 23, red arrow), and a putative cleavage site (blue arrow). Seven conserved cystine residues (underlined) are located in the cystine-knot domain. 
A
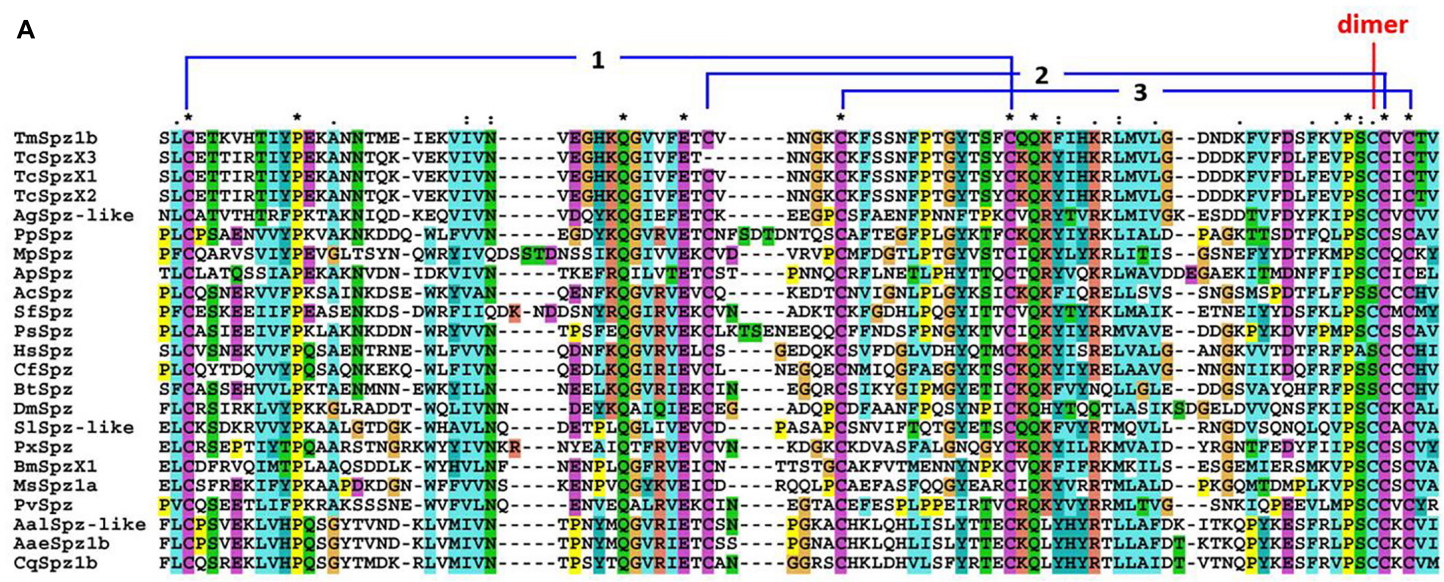

B<smiles>CC(Cl)(Cl)[Ge]</smiles>

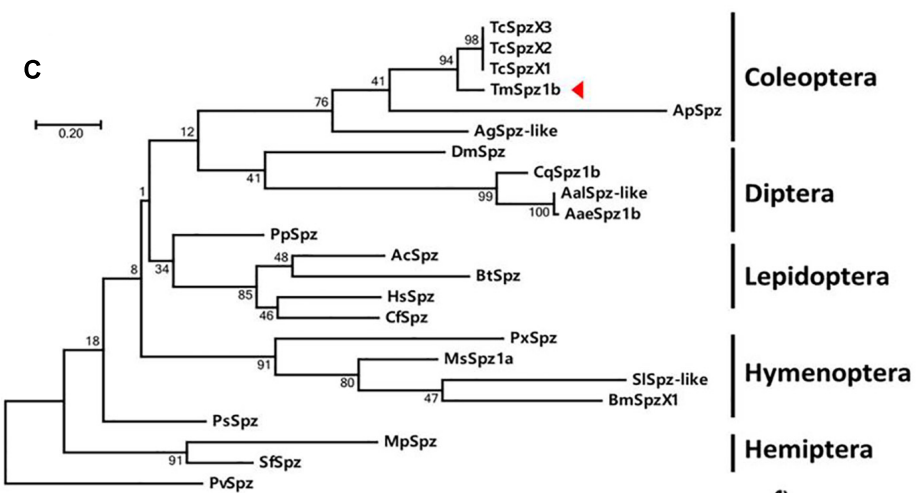

D

\begin{tabular}{|c|c|c|c|c|c|c|c|c|c|c|c|c|c|c|c|c|c|c|c|c|c|c|}
\hline & 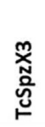 & 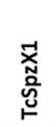 & 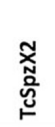 & 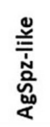 & $\begin{array}{l}\text { 음 } \\
\text { 은 }\end{array}$ & 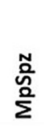 & $\begin{array}{l}\frac{0}{2} \\
\frac{2}{\alpha}\end{array}$ & 苍 & 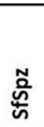 & 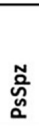 & 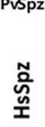 & 总 & 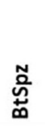 & 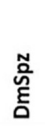 & 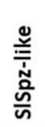 & $\begin{array}{l}\text { o } \\
\text { ஸे }\end{array}$ & 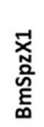 & 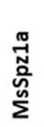 & 誉 & 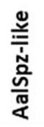 & 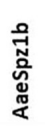 & 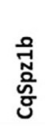 \\
\hline nSpz1b & 85 & 85 & 85 & 49 & 41 & 40 & 40 & 37 & 37 & 36 & 36 & 36 & 35 & 33 & 33 & 31 & 29 & 29 & 28 & 27 & 27 & 27 \\
\hline SpzX3 & & 100 & 100 & 49 & 41 & 38 & 42 & 36 & 34 & 37 & 36 & 40 & 34 & 34 & 30 & 33 & 24 & 29 & 27 & 26 & 26 & 27 \\
\hline SpzX1 & & & 100 & 49 & 41 & 40 & 42 & 36 & 35 & 37 & 36 & 40 & 34 & 34 & 30 & 34 & 25 & 29 & 28 & 27 & 27 & 28 \\
\hline SpzX2 & & & & 49 & 41 & 40 & 42 & 36 & 35 & 37 & 36 & 40 & 34 & 34 & 30 & 34 & 25 & 29 & 28 & 27 & 27 & 28 \\
\hline gSpz-like & & & & & 40 & 29 & 33 & 32 & 33 & 39 & 32 & 34 & 30 & 42 & 25 & 32 & 32 & 27 & 30 & 32 & 31 & 27 \\
\hline Spz & & & & & & 37 & 35 & 49 & 42 & 55 & 49 & 52 & 42 & 38 & 37 & 39 & 34 & 44 & 36 & 40 & 40 & 39 \\
\hline pSpz & & & & & & & 32 & 35 & 50 & 38 & 28 & 33 & 32 & 33 & 27 & 28 & 28 & 28 & 35 & 27 & 27 & 31 \\
\hline Spz & & & & & & & & 27 & 31 & 35 & 30 & 30 & 26 & 31 & 24 & 27 & 20 & 26 & 31 & 33 & 34 & 31 \\
\hline Spz & & & & & & & & & 37 & 48 & 55 & 54 & 49 & 32 & 38 & 33 & 33 & 34 & 38 & 32 & 32 & 32 \\
\hline spz & & & & & & & & & & 44 & 36 & 35 & 34 & 35 & 28 & 34 & 31 & 32 & 37 & 37 & 35 & 37 \\
\hline$p z$ & & & & & & & & & & & 45 & 47 & 43 & 33 & 34 & 39 & 33 & 41 & 45 & 38 & 38 & 40 \\
\hline Spz & & & & & & & & & & & & 53 & 48 & 32 & 33 & 37 & 29 & 33 & 35 & 39 & 39 & 36 \\
\hline Spz & & & & & & & & & & & & & 48 & 33 & 34 & 37 & 29 & 32 & 33 & 38 & 38 & 39 \\
\hline spz & & & & & & & & & & & & & & 32 & 37 & 30 & 27 & 29 & 36 & 34 & 33 & 34 \\
\hline nSpz & & & & & & & & & & & & & & & 32 & 31 & 24 & 31 & 24 & 37 & 37 & 38 \\
\hline pz-like & & & & & & & & & & & & & & & & 33 & 41 & 47 & 33 & 26 & 26 & 28 \\
\hline Spz & & & & & & & & & & & & & & & & & 38 & 38 & 29 & 32 & 31 & 31 \\
\hline ISpzX1 & & & & & & & & & & & & & & & & & & 46 & 30 & 25 & 25 & 27 \\
\hline Spz1a & & & & & & & & & & & & & & & & & & & 32 & 32 & 32 & 34 \\
\hline$p z$ & & & & & & & & & & & & & & & & & & & & 33 & 32 & 33 \\
\hline Spz-like & & & & & & & & & & & & & & & & & & & & & 96 & 76 \\
\hline Spz1b & & & & & & & & & & & & & & & & & & & & & & 76 \\
\hline
\end{tabular}

FIGURE 2 | Multiple sequence alignment and phylogenetic analyses of Spätzle proteins. (A) Multiple sequence alignment of the spätzle domain in Spätzle proteins. A high degree of conservation is evident in the spätzle domain. The conserved cysteine residues forming the three disulfide bonds are shown. The cysteine involved in the dimer formation is also denoted. (B) Deduced cystine-knot domain of TmSpz1b. The six cystine residues form three disulfide bridges, and the one extra cystine residue may interact with the other active form of Spätzle protein. (C) Phylogenetic tree of Spätzle proteins. TmSpz1b is located on the same branch occupied by TcSpzX1, TcSpzX2, and TcSpzX3. (D) Percentage identity of TmSpz1b with its orthologs. The Penaeus vannamei spätzle protein (PvSpz) sequence 
FIGURE 2 | was used as the outgroup. TmSpz1b, Tenebrio molitor Spätzle-1b; AgSpzlike, Anoplophora glabripennis protein spaetzle-like (XP_018564206.1); ApSpz, Agrilus planipennis protein spaetzle (XP_018334006.1); TcSpzX3, Tribolium castaneum PREDICTED: protein spaetzle isoform X3 (XP_015840683.1); TcSpzX1, Tribolium castaneum PREDICTED: protein spaetzle isoform X1 (XP_008201187.1); BtSpz, Bombus terrestris protein spaetzle (XP_003402363.1); HsSpz, Harpegnathos saltator protein spaetzle (XP_011149648.1); CfSpz, Camponotus floridanus protein spaetzle (XP_011256297.1); AcSpz, Apis cerana cerana Protein spaetzle (PBC29562.1); PsSpz, Plautia stali protein spaetzle (BBE08127.1); MpSpz, Myzus persicae protein spaetzle-like (XP_022173331.1); PpSpz, Pristhesancus plagipennis secreted Spaetzle-like protein (ATU82783.1); SfSpz, Sipha flava protein spaetzle (XP_025420977.1); CqSpz1b, Culex quinquefasciatus spätzle 1B (XP_001864596.1); AalSpz-like, Aedes albopictus protein spaetzle-like (XP_029718352.1); AaeSpz1b, Aedes aegypti spaetzle1B precursor (NP_001350875.1); DmSpz, Drosophila melanogaster spätzle (ABM21577.1); PxSpz, Papilio xuthus Protein spaetzle (KPJ02943.1); S/Spz-like, Spodoptera litura protein spaetzle-like (XP_022825571.1); BmSpzX1, Bombyx mori spätzle-1 isoform X1 (XP_021206899.1); MsSpz1a, Manduca sexta Spz1A (ACU68553.1); PvSpz, Penaeus vannamei protein spaetzle (ROT72693.1).
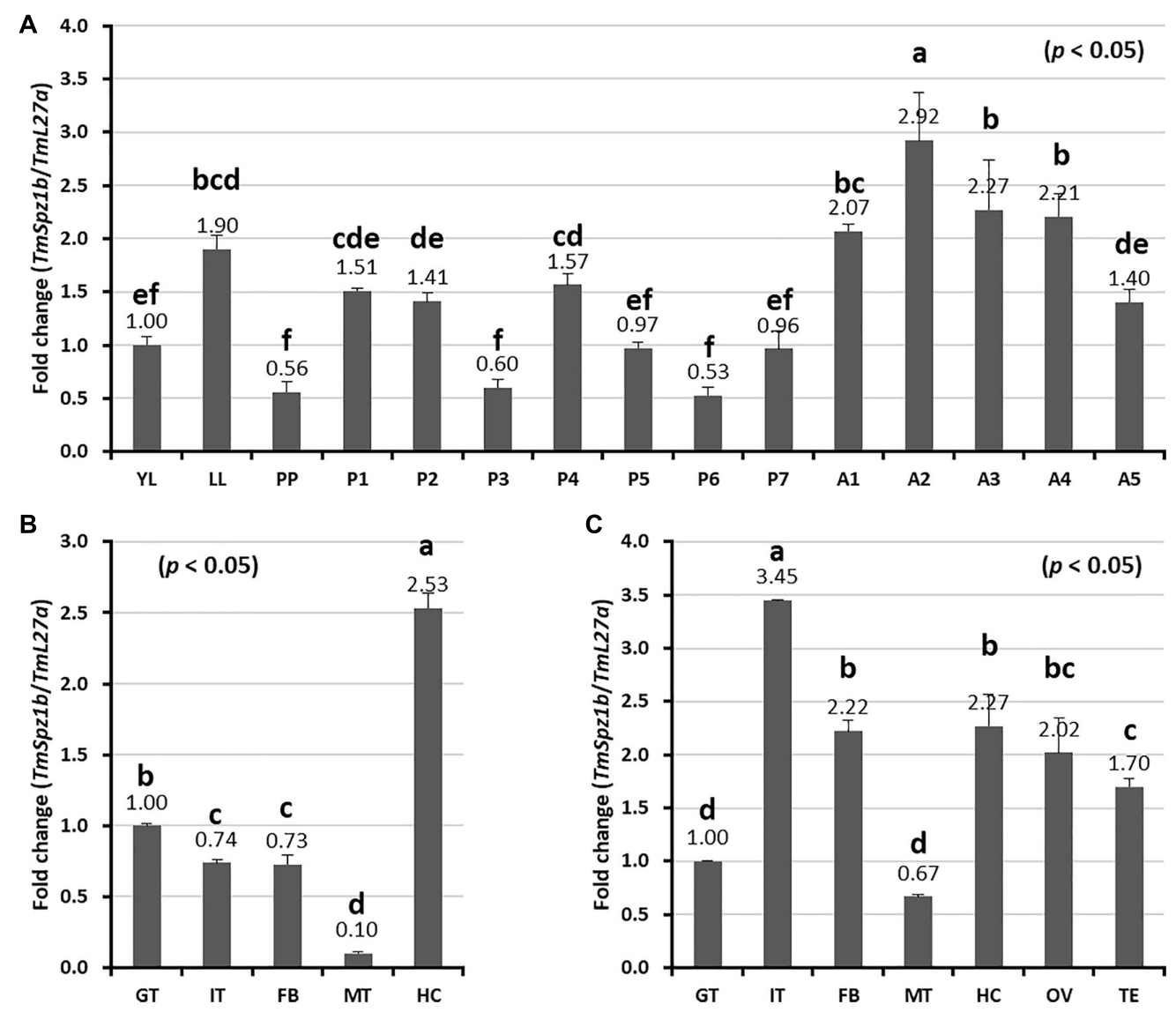

FIGURE 3 | Expression profiles of TmSpz1b mRNA during development and in tissues of T. molitor assessed by real-time PCR. (A) Developmental expression patterns of TmSpz1b mRNA. YL, young larvae (10th-12th instar); LL, late instar larvae; PP, pre-pupae; P1-P7, 1- to 7-day-old pupae; and A1-A5, 1- to 5-day-old adults. Tissue-specific expression profiles of TmSpz1b were examined in late instar larvae (B) and adults (C). IT, integument; GT, gut; FB, fat body; HC, hemocytes; MT, Malpighian tubules; OV, ovary; and TS, testis. Total RNAs extracted from developmental stages and different tissues were reverse transcribed to cDNAs to serve as templates. Vertical bars represent mean \pm standard error of three biological replicates. One-way ANOVA and Tukey's multiple range tests at $95 \%$ confidence leve $(p<0.05)$ were performed and used to determine the level of significant differences. The graphs indicated by the same letter are not significantly different in Tukey's multiple range test $(p<0.05)$.

except Hymenopteran insects (Figure 2A). Domain analysis of TmSpzib also determined a conserved cystine-knot domain harboring seven cystine residues and a putative cleavage site. In addition, six cystine resides formed three disulfide bridges, with the remaining cystine residue perhaps involved in the formation of an active spätzle dimer (Figure 2B). Phylogenetic analysis indicated that TmSpzlb was located on the same branch with coleopteran insects, including the T. castaneum protein spätzle isoforms X1, X2, and X3 (Figure 2C). Approximately 85\% sequence identity was shared between TmSpzlb and TcSpzX1, $\mathrm{X} 2$, and $\mathrm{X} 3$ (Figure 2D).

\section{Developmental and Tissue-Specific Expression Patterns of TmSpz1b}

To understand the basic expression patterns of TmSpz1b, developmental and tissue-specific expression profiles were investigated by qRT-PCR analysis. TmSpz1b was highly expressed 


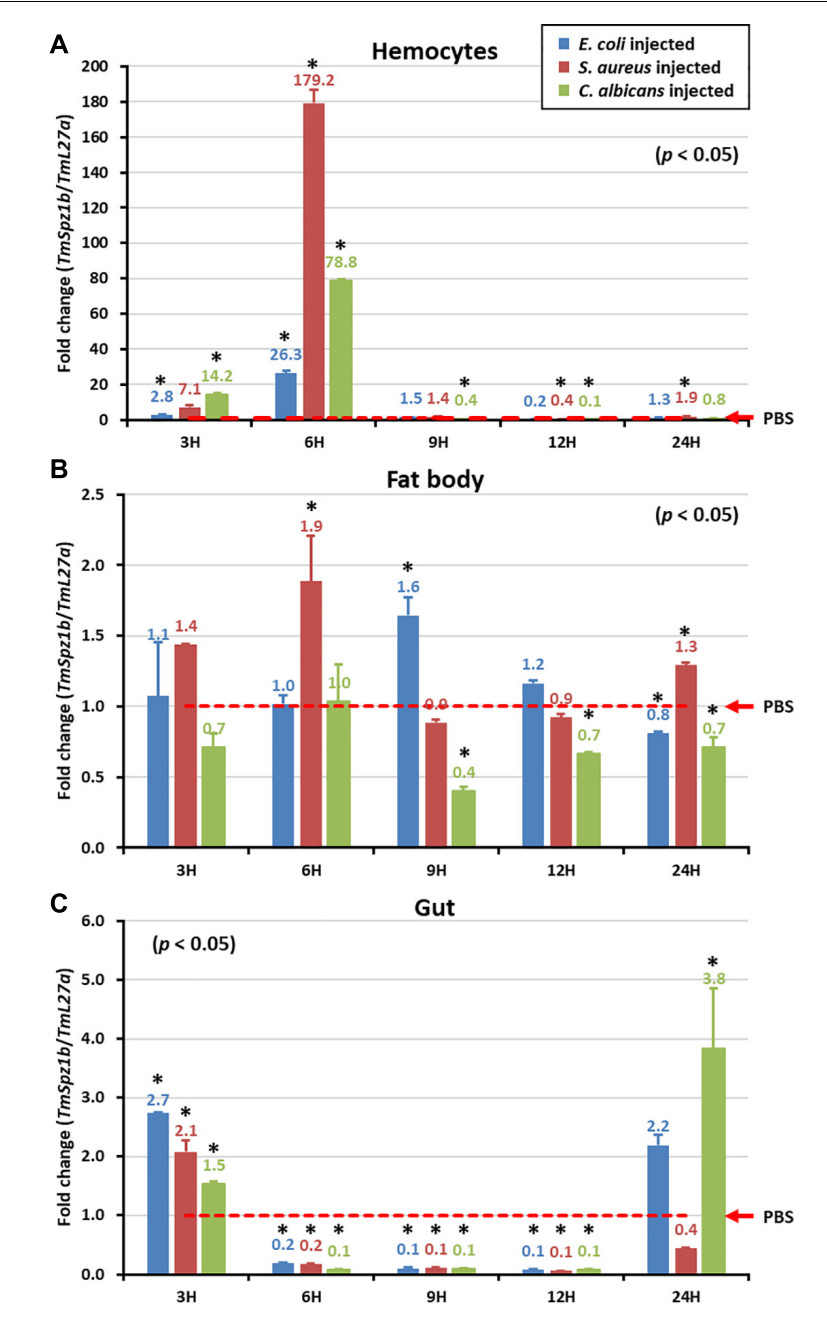

FIGURE 4 | Temporal expression patterns of TmSpz1b in three immune organs following microbial challenge. After injection of E. coli, S. aureus, or C. albicans, three immune organs including hemocytes (A), fat bodies (B), and the gut (C) were dissected and collected at 3, 6, 9, 12, and $24 \mathrm{~h}$. TmSpz1b expression was analyzed by qRT-PCR. PBS-treated group was used as the mock control. The expression level of TmSpz-like mRNA in the mock control group was normalized to 1. Vertical bars depict the mean \pm S.E. of three biological replicates. Significant differences $(P<0.05)$ between the experimental and control group are indicated by asterisks $\left({ }^{*}\right)$.

at the 2-day-old adult stage. The lowest expression was observed in the prepupal and 3- and 6-day-old pupal stages (Figure 3A). In general, the expression of TmSpz1b mRNA was greater in adults and in the late-larval stage. In addition, tissue-specific expression patterns of TmSpzlb were examined in late instar larvae (Figure 3B) and 5-day-old adults (Figure 3C). The results indicated that $T m S p z 1 b$ is highly expressed in the hemocytes (2.5-fold) of late instar larvae and integument (approximately 3.5-fold) of 5-day-old adults.

\section{Induction Patterns of TmSpz1b}

To elucidate the putative role of TmSpz1b in Tenebrio innate immunity, temporal expression patterns of $T m S p z 1 b$ against microbial challenges were investigated in different immune organs. E. coli $\left(1 \times 10^{6}\right.$ cells $\left./ \mu \mathrm{l}\right), S$. aureus $\left(1 \times 10^{6}\right.$ cells $\left./ \mu \mathrm{l}\right)$, and $C$. albicans $\left(5 \times 10^{4}\right.$ cells $\left./ \mu \mathrm{l}\right)$ were injected into $T$. molitor 10 th to 12 th instar larvae. Three immune organs (hemocytes, fat bodies, and the gut), were collected at different times $(3,6,9,12$, and $24 \mathrm{~h}$ ). In hemocytes, TmSpz1b was dramatically induced at $6 \mathrm{~h}$ after injection of $E$. coli (approximately 30-fold), S. aureus (approximately 180-fold), and C. albicans (80-fold) (Figure 4A). $T m S p z 1 b$ mRNA expression was drastically reduced at later time points after a dramatic increase at $6 \mathrm{~h}$ post-infection. However, $T m S p z 1 b$ expression was not strongly induced in the fat bodies and the gut. Interestingly, in the gut, TmSpz1b expression was significantly decreased at 6,9 , and $12 \mathrm{~h}$ following the injection of microorganisms, compared to that in the PBS injected control group (Figures 4B,C).

\section{Knockdown of TmSpz1b Decreased Larval Survivable Following Microbial Challenges}

To assess the function of highly expressed TmSpz1b in hemocytes, the effects of TmSpz1b RNAi on larval survivable following microbial challenges were investigated. Initially, TmSpz1b dsRNA ( $1 \mu \mathrm{g} /$ larva) was injected into $T$. molitor larvae. The knockdown ratio was investigated by qRT-PCR analysis. A decrease of TmSpz1b expression of approximately 80\% (0.2fold) was observed following injection of TmSpz1b-specific dsRNA, compared to that in the dsEGFP-treated group (1.0-fold) at 2 days post-injection (Figure 5A).

Following injection of E. coli, S. aureus, and C. albicans into TmSpz1b-silenced T. molitor larvae, the survival of the larvae was monitored for 10 days. Interestingly, larval survivability upon E. coli challenge, but not upon challenge with $S$. aureus and $C$. albicans, was significantly decreased by knockdown of TmSpz1b, compared to that in the dsEGFP-treated group (Figures 5B-D). In E. coli treated TmSpz1b-silenced larvae, survival was reduced to $40 \%$ and was significantly different from the dsEGFP-treated larvae up to 10 days after infection. The survival of $S$. aureus- and C. albicans-infected TmSpz1b-silenced larvae was reduced by nearly $80 \%$ but was not significantly different from the dsEGFP-treated groups.

\section{Effects of TmSpz1b RNAi on Expression of 14 Antimicrobial Peptide Genes}

Next, to determine the mechanism of action of TmSpz1b gene in the humoral immunity of $T$. molitor, the expression of 14 AMP genes was investigated by qRT-PCR analysis after microbial challenge of the TmSpz1b-silenced T. molitor larvae. In hemocytes, the expression levels of seven of the 14 AMP genes were positively regulated (i.e., downregulated in TmSpz1bsilenced individuals) (Figure 6A). TmTene1 was decreased by $56 \%$ in E. coli, $29.4 \%$ in S. aureus, and $72.7 \%$ in C. albicans (Figure 6A). The respective decreases for TmTene3 were 83, 75, and 76\% (Figure 6C). The respective decreases for TmAtt1a were 82,83 , and $88 \%$ (Figure $6 \mathrm{E}$ ). The respective decreases for $T m A t t 1 b$ were 30, 52, and 50\% (Figure 6F). The respective decreases for TmColeA were 85, 86, and 88\% (Figure 6H). 

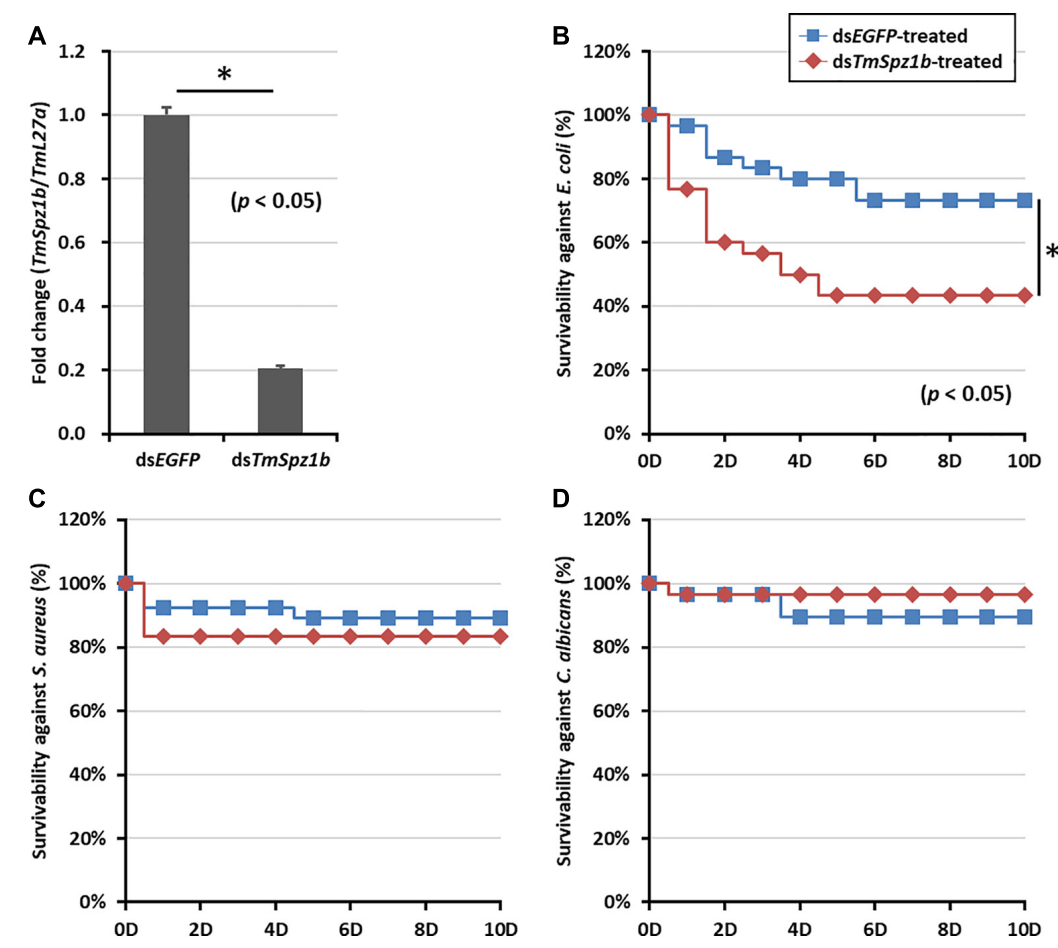

FIGURE 5 | Effects of TmSpz1b gene-silencing on larval survival upon microbial challenges. (A) Validation of RNAi in dsTmSpz1b treated larvae compared with that in dsEGFP-treated larvae. TmSpz1b expression was decreased by approximately $80 \%$ following the injection of TmSpz1b-specific dsRNAs, compared to that in the dsEGFP-treated group. E. coli (B), S. aureus (C), and C. albicans (D) were injected into TmSpz1b-silenced T. molitor larvae, and larval survival was monitored for 10 days. The dsEGFP-treated groups were used as controls. Survival of larvae infected with E. coli, but not $S$. aureus and C. albicans, was significantly decreased by $T m S p z 1 b$ knockdown. The data are an average of three biologically independent replicate experiments. Asterisks indicate significant differences between dsTmSpz4and dsEGFP-injected groups $(P<0.05)$. Statistical analysis of survival analysis was carried out based on Kaplan-Meier plots (log-rank chi-square test; * $P<0.05)$.

The respective decreases for TmColeB were 91, 81, and $83 \%$ (Figure 6I). Finally, the respective decreases for TmDef-like were 53,46 , and $77 \%$ (Figure $6 \mathbf{K}$ ). In fat bodies, the expression of five AMP genes was positively regulated in TmSpz1b-silenced larvae (Figure 7). The respective decreases in E. coli, S. aureus, and C. albicans were 82, 83, and 88\% TmAtt1a (Figure 7E); 30, 52, and $50 \%$ for TmAtt $1 b$ (Figure $7 F$ ); 85, 86, and $88 \%$ for TmColeA (Figure 7H); 90, 82, and 83\% for TmColeB (Figure 7I); and 93, 0, and $70 \%$ for TmTLP1 (Figure 7L).

In the gut, significantly decreased expression was detected in only one AMP gene (Figure 8). TmColeB was decreased by $68 \%$ in E. coli, $67 \%$ in S. aureus, and $96 \%$ in C. albicans by TmSpz1b RNAi (Figure 8I). Further, to delineate the regulatory role of TmSpz1b in the Toll/IMD signaling cascade mechanism, we studied the transcriptional regulation of NF- $\kappa \mathrm{B}$ factors such as TmDorX2 (Toll pathway) and TmRelish (IMD pathway). The transcriptional levels of TmDorX2 and TmRelish after TmSpz1b silencing and challenge of E. coli, S. aureus, and C. albicans is shown in Figure 9. There was a positive regulation of TmDorX2 transcripts upon TmSpz1b silencing after all microorganisms challenge in hemocytes and fat body tissue while in gut it was observed in case of E. coli and C. albicans infection (Figure 9A). In $E$. coli challenged individuals, maximum downregulation of TmDorX2 transcripts were observed under TmSpz1b silencing conditions. The TmRelish transcripts were mostly found to be negatively regulated in $T m S p z 1 b$ silenced individuals except in case of $E$. coli infection in hemocytes and fat body tissue (Figure 9B). A pertinent observation was that TmSpz1b released from the hemocytes positively regulated TmTene1, TmAtt1a, TmAtt1b, TmColeA, TmColeB, and TmDef-like in the hemocytes and TmAtt1a, TmAtt1b, TmColeA, TmColeB, and TmTLP2 in the fat bodies. Activity of these genes could possibly kill E. coli in hemocoel. Thus, survival of T. molitor larvae was improved (Figure 10A). On the contrary, TmSpzlb silencing had no impact on the resistance to $S$. aureus and C. albicans infections in T. molitor larvae (Figure 10B). These observations suggest that $T m S p z 1 b$ is required to confer antibacterial defense against Gram-negative bacteria and not Gram-positive bacteria and fungi, by regulation of AMPs in hemocytes and fat bodies.

\section{DISCUSSION}

Spätzle protein has an important role in dorsal-ventral polarity in Drosophila and invertebrate development. Its immunological role has been characterized in insects as well as aquatic invertebrates (Imler and Hoffmann, 2002). The endogenous Drosophila spätzle protein is critical for the activation of the Toll pathway by direct binding to Toll receptors. In the present study, a novel spätzle isoform (TmSpzlb) involved in the T. molitor innate immunity 

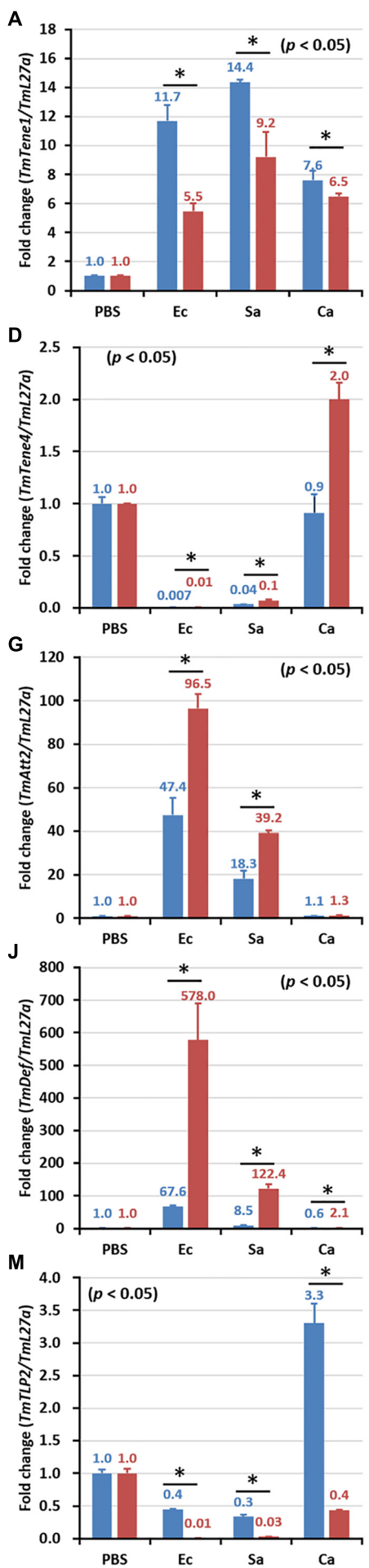
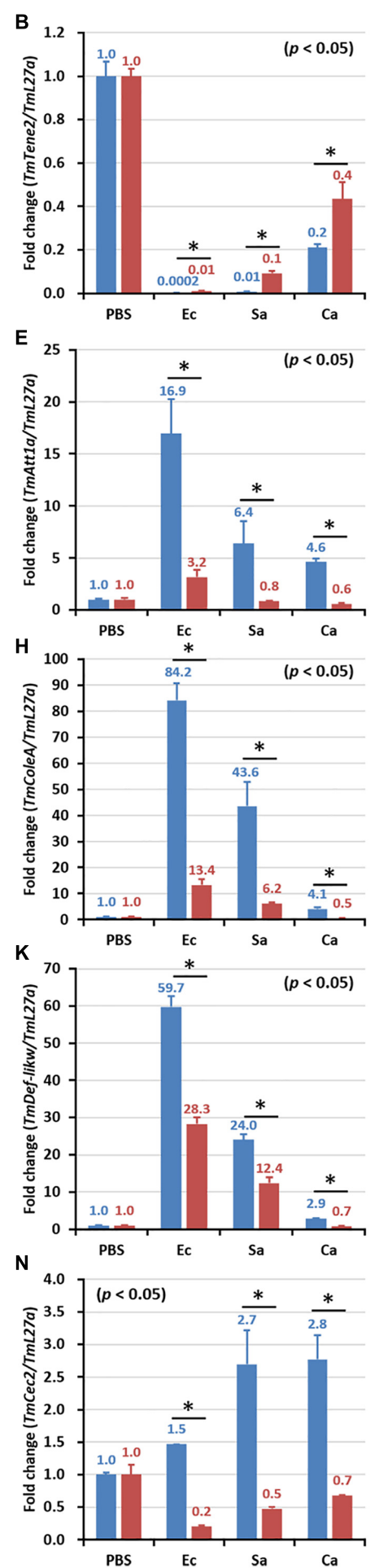
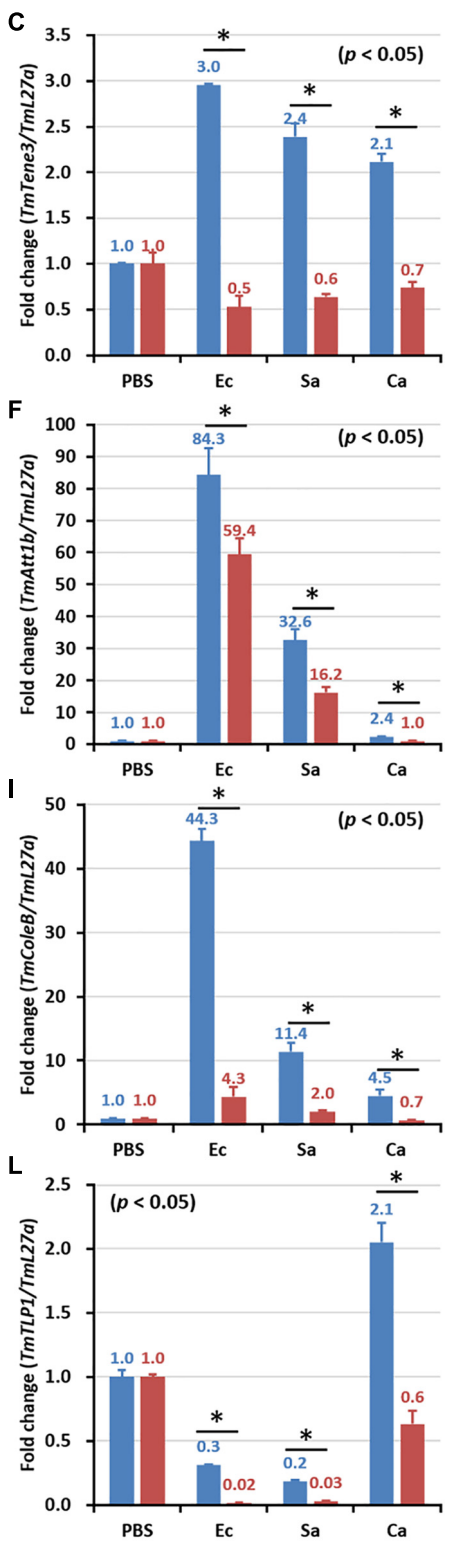

FIGURE 6 | Effects of TmSpz1b gene-silencing on the expression of 14 AMP genes in response to pathogen injection in hemocytes. E. coli, S. aureus, and C. albicans were injected into dsTmSpz1b-treated T. molitor larvae. The expression levels of 14 AMP genes were determined at $24 \mathrm{~h}$ post injection, by qRT-PCR. In hemocytes, seven AMP genes (TmTenecin 1 and 3, TmAttacin1a, and 1b, TmColeoptericin 1 and 2, and TmDefensin2) were significantly decreased by TmSpz1b RNAi. dsEGFP was injected as a negative control, and TmL27a was used as an internal control. All experiments were performed in triplicate. Asterisks indicate significant differences between dsTmSpz1b and dsEGFP-treated groups when compared by Student's t-test $(P<0.05)$. TmTene1 (A; TmTeneecin-1), TmTene2 (B; TmTenecin-2). TmTene3 (C; TmTenecin-3), TmTene4 (D; TmTenecin-4), TmAtt1a (E; TmAttacin-1a), TmAtt1b (F; TmAttacin-1b), TmAtt2 (G; TmAttacin-2), TmCole1 (H; TmColeoptericin-1), TmCole2 (I; TmColeoptericin-2), TmDef1 (J; TmDefensin-1), TmDef2 (K; TmDefensin-2), TmTLP1 (L; TmThaumatin-like protein-1), TmTLP2 (M; TmThaumatin-like protein-2), TmCec2 (N; TmCecropin-2). 

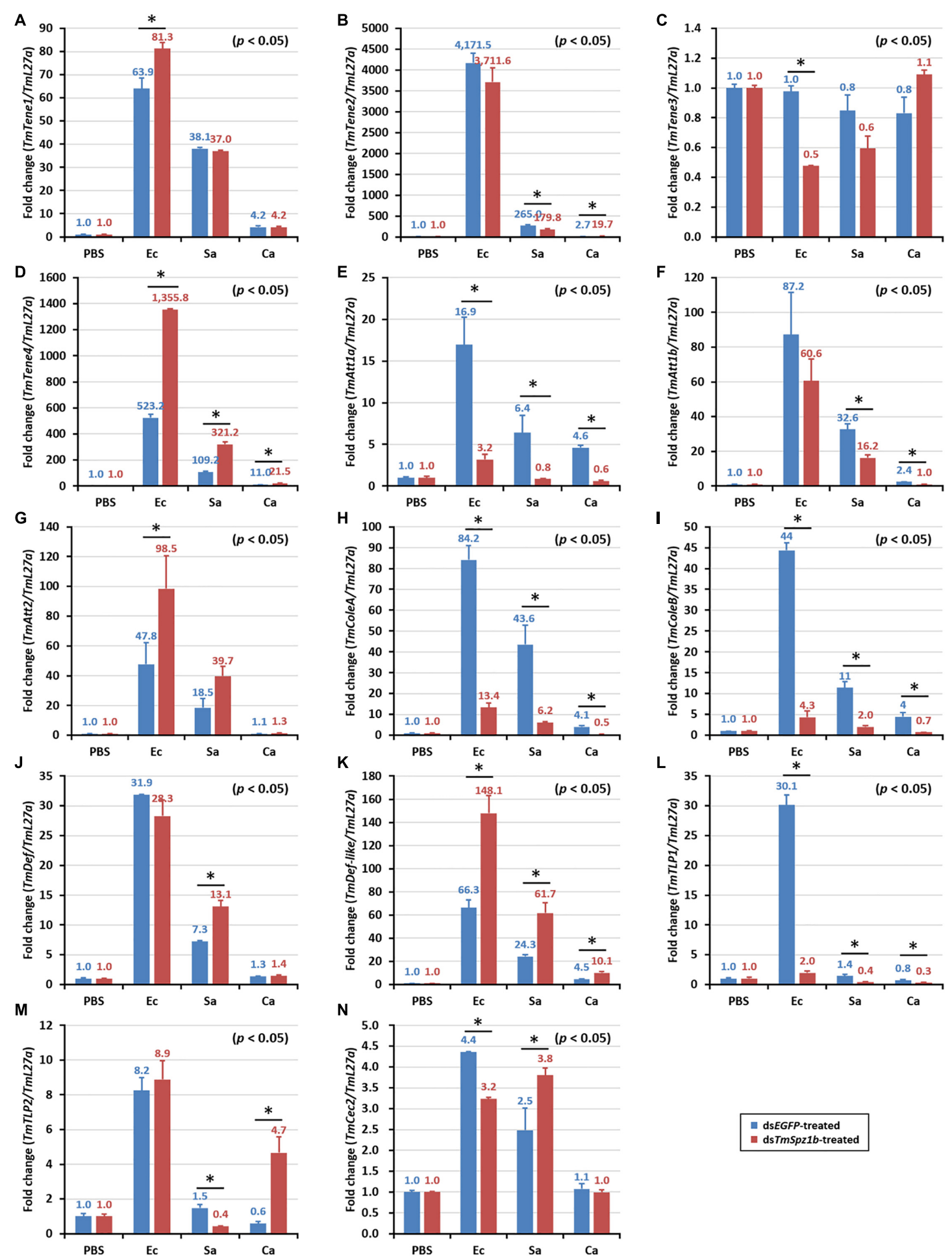

FIGURE 7 | Effects of TmSpz1b gene-silencing on the expression of 14 AMP genes in response to pathogen injection in fat body. E. coli, S. aureus, and C. albicans were injected into dsTmSpz1b-treated T. molitor larvae. The expression levels of 14 AMP genes were determined at $24 \mathrm{~h}$ post injection, by qRT-PCR. In fat bodies, five AMP genes (TmAttacin1a, and 1b, TmColeoptericin1 and 2, and TmTLP1) were significantly decreased by TmSpz1b RNAi. dsEGFP was injected as a negative control and TmL27a was used as an internal control. All experiments were performed in triplicate. Asterisks indicate significant differences between dsTmSpz1b and dsEGFP-treated groups when compared by Student's t-test $(P<0.05)$. TmTene1 (A; TmTeneecin-1), TmTene2 (B; TmTenecin-2). TmTene3 (C; TmTenecin-3), TmTene4 (D; TmTenecin-4), TmAtt1a (E; TmAttacin-1a), TmAtt1b (F; TmAttacin-1b), TmAtt2 (G; TmAttacin-2), TmCole1 (H) TmColeoptericin-1), TmCole2 (I; TmColeoptericin-2), TmDef1 (J; TmDefensin-1), TmDef2 (K; TmDefensin-2), TmTLP1 (L; TmThaumatin-like protein-1), TmTLP2 (M; TmThaumatin-like protein-2), TmCec2 (N; TmCecropin-2). 

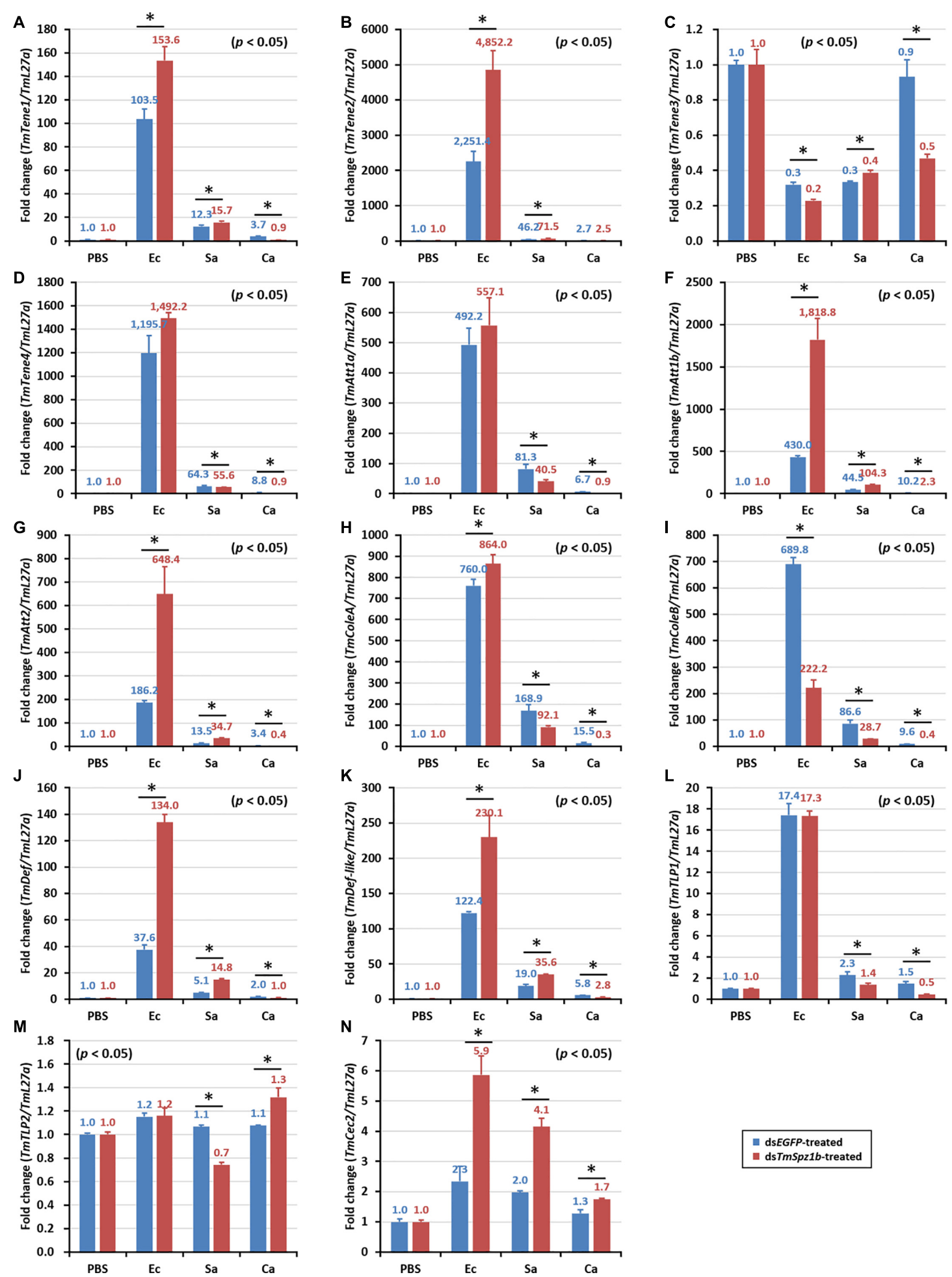

dsEGFP-treated

dsTmSpz1b-treated

FIGURE 8 | Effects of TmSpz1b gene-silencing on the expression of 14 AMP genes in response to pathogen injection in the gut. E. coli, S. aureus, and C. albicans were injected into dsTmSpz1b-treated T. molitor larvae. The expression levels of 14 AMP genes were determined at 24-post injection, by qRT-PCR. In the gut, only one AMP gene (TmColeoptericin2) was significantly decreased by TmSpz1b RNAi. dsEGFP was injected as a negative control, and TmL27a was used as an internal control. All experiments were performed in triplicate. Asterisks indicate significant differences between dsTmSpz1b and dsEGFP-treated groups when compared by Student's t-test $(P<0.05)$. TmTene1 (A; TmTeneecin-1), TmTene2 (B; TmTenecin-2). TmTene3 (C; TmTenecin-3), TmTene4 (D; TmTenecin-4), TmAtt1a (E; TmAttacin-1a), TmAtt1b (F; TmAttacin-1b), TmAtt2 (G; TmAttacin-2), TmCole1 (H; TmColeoptericin-1), TmCole2 (I; TmColeoptericin-2), TmDef1 (J; TmDefensin-1), TmDef2 (K; TmDefensin-2), TmTLP1 (L; TmThaumatin-like protein-1), TmTLP2 (M; TmThaumatin-like protein-2), TmCec2 (N) TmCecropin-2). 

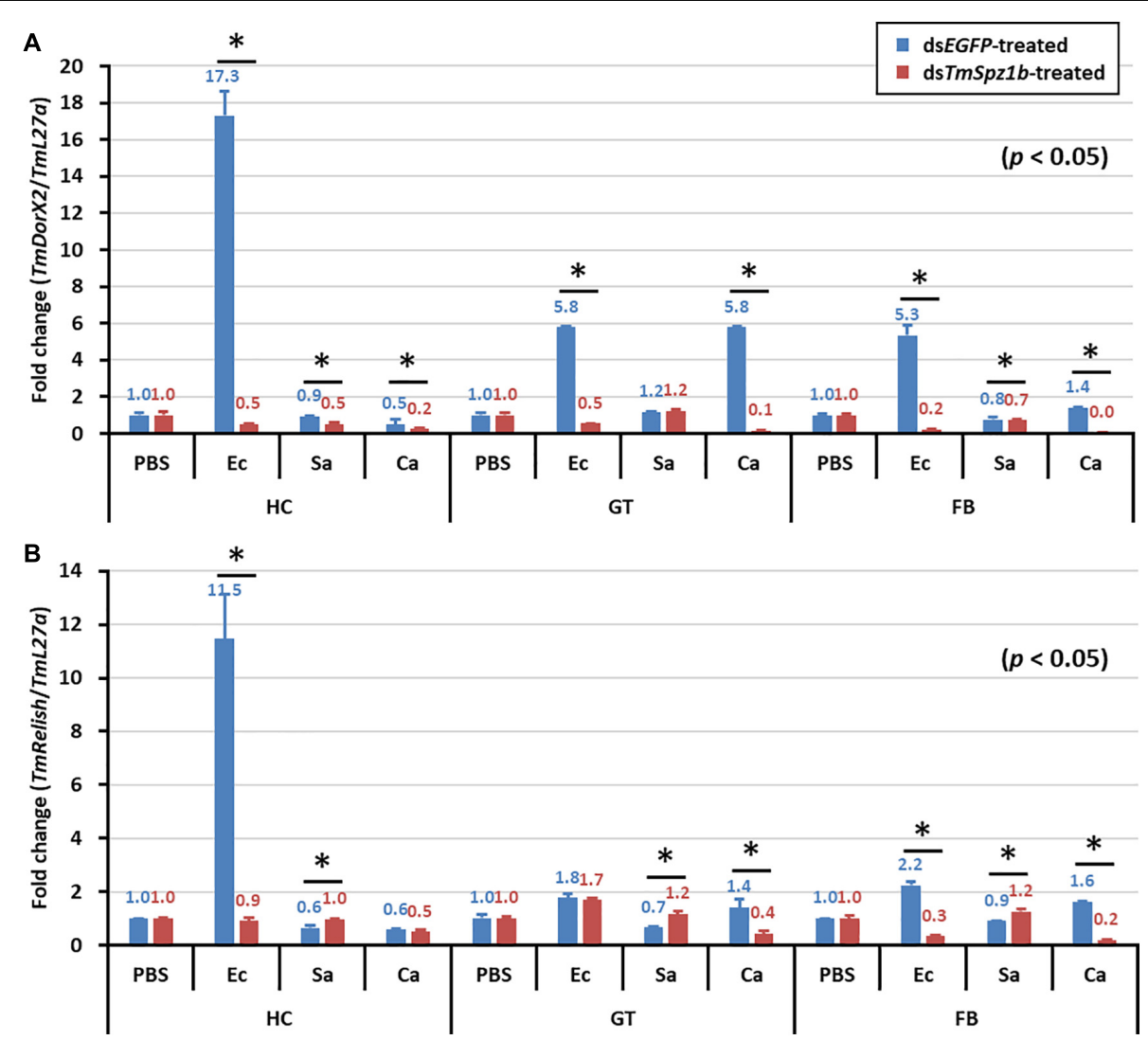

FIGURE 9 | Effect of TmSpz-1b silencing on the transcriptional regulation of NF-кB genes after injections of E. coli, S. aureus, and C. albicans. mRNA expression levels of the NF-кB genes viz. TmDorX2 (A), and TmRelish (B) have been investigated by RT-qPCR. Larvae were injected with dsEGFP as a negative control, and $\mathrm{TmL27a}$ expression was assessed as an internal control. All experiments were performed in triplicate. Asterisks indicate significant differences in NF-kB gene expression between the dsTmSpz-like- and dsEGFP-treated groups when compared by Student's $t$-test $(P<0.05)$.

was reported, that causes the sequestration of Gram-negative bacteria by the regulatory action of AMPs and enhances the survival of T. molitor larvae. Silencing of TmSpz1b led to the positive regulation of AMPs in hemocytes and fat bodies of T. molitor larvae, suggesting the requirement of TmSpz1b in microbial killing (specifically against E. coli) due to action of AMPs in the hemocoel.

Initially, the TmSpz1b gene was identified by bioinformatics analysis from T. molitor RNA sequencing database. The ORF sequence was confirmed by cloning and sequencing. Domain analysis of TmSpz1b indicated a signal peptide region (indicating secretion to hemocoel), one putative cleavage site, and one cystine-knot domain composed of 93 amino acid residues. A previous study structurally characterized the disulfide-linked cystine-knot dimer by crystallization (Hoffmann et al., 2008b). In addition, the same authors described the cleavage of the pre-form of spätzle protein in D. melanogaster by trypsin, and a cystineknot domain with seven conserved cysteine residues in the C106 active form of spätzle (Hoffmann et al., 2008a). The five homologs of spätzle (spz2 - 6), which had a neurotrophin-like cystineknot domain, were identified by blast analysis with the Drosophila genomic and transcriptomic database (Parker et al., 2001). The cystine-knot domain and the specific cysteine residues involved in the formation of disulfide bridges have also been identified in spätzle proteins of B. mori and M. sexta (Wang et al., 2007; An et al., 2010). Unlike other insect spätzle proteins, the spätzle protein in Chinese oak silkworm, A. pernyi (ApSpz) contains a cystine-knot domain with only two conserved cysteine residues (Sun et al., 2016). The T. molitor spätzle protein isoforms such as TmSpz4 and TmSpz6 also contain a cysteine knot domain in their C-terminus with conserved cysteine residues forming disulfide bridges (Edosa et al., 2020a,b). In addition, examination of the spätzle proteins in aquatic invertebrates, such as shrimp and clam, has revealed a conserved cystine-knot domain. The deduced amino acid sequence of Spätzle-like protein identified from the expressed sequence tag of Chinese shrimp, F. chinensis $(F c S p z)$, includes a signal peptide region and a cystine-knot domain with seven cysteine residues (Shi et al., 2009). Spätzle proteins from other shrimp, such as Penaeus monodon (PmSpz1) and Litopenaeus vannamei ( $\mathrm{LvSpz} 4)$, as well as the first mollusk spätzle homolog gene identified from $P$. undulate, also include a cystine-knot domain with seven cysteine residues (Yu et al., 2015; Boonrawd et al., 2017; Yuan et al., 2017). A signal peptide region that is promiscuous in all spätzle proteins enables its transport through cell membranes and secretion to the hemocoel. Consistent with these previous studies, our results indicated that 


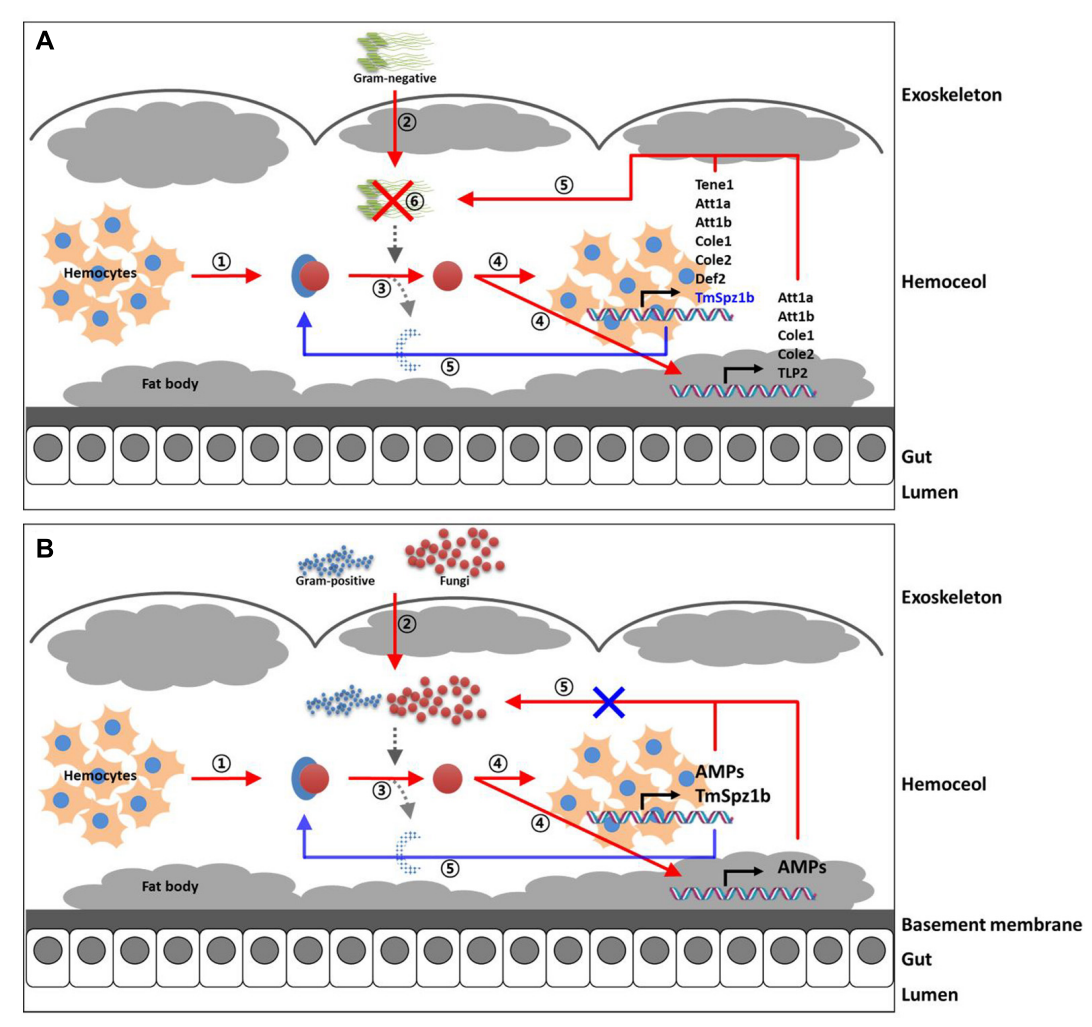

FIGURE 10 | Proposed immunological function of TmSpz1b in innate immune response to microbial challenges. The immunological functions of $T m S p z 1 \mathrm{~b}$ against E. coli (A), S. aureus, and C. albicans (B) are separately proposed. (A) TmSpz1b influences the transcriptional regulation of seven and five AMP genes in hemocytes and fat body of T. molitor larvae in killing the Gram-negative pathogen E. coli. (B) TmSpz1b does not affect the resistance to Gram-positive and fungal infections in T. molitor larvae due to non-regulation of AMP genes.

TABLE 2 | Important AMP genes predicted by our recent results.

\begin{tabular}{|c|c|c|c|c|c|}
\hline Gene name & & $T m S p z 1 b$ & & TmCactin & TmToll-7 \\
\hline $\begin{array}{c}\text { Genes active against } \\
\text { E. coli }\end{array}$ & $\begin{array}{c}\text { TmTene1 } \\
\text { TmDef-like } \\
\text { TmColeA } \\
\text { TmColeB } \\
\text { TmAtt-1a } \\
\text { TmAtt-1b }\end{array}$ & $\begin{array}{c}\text { TmColeA } \\
\text { TmColeB } \\
\text { TmAtt-1a } \\
\text { TmAtt-1b } \\
\text { TmTLP1 }\end{array}$ & TmColeB & $\begin{array}{c}\text { TmTene1 } \\
\text { TmTene4 } \\
\text { TmDef } \\
\text { TmDef-like } \\
\text { TmColeA } \\
\text { TmColeB } \\
\text { TmAtt-1b }\end{array}$ & $\begin{array}{c}\text { TmTene1 } \\
\text { TmDef } \\
\text { TmDef-like } \\
\text { TmColeA } \\
\text { TmAtt-2 }\end{array}$ \\
\hline References & - & - & - & Jo et al., 2017 & Park et al., 2019 \\
\hline
\end{tabular}

Bold texts mean some AMPs overlapped by different RNAi.

TmSpz1b may be secreted from the cells to the hemocoel and that serine protease (the Spätzle processing enzyme) may activate TmSpzib. In Drosophila, Persephone in response to danger signals and damage associated molecular patterns (DAMPs) are also responsible for cleaving spätzle and seem to be important in differentiating harmful microbes from commensals (Shaukat et al., 2015; Issa et al., 2018). Silencing of Spätzle processing enzyme in Drosophila mutants leads to impaired immunity against the Gram-positive bacterium Enterococcus faecalis and not the Gram-negative bacterium Pseudomonas aeruginosa, suggesting a role of Spätzle processing enzyme in the Toll pathway (Mulinari et al., 2006). Seven cysteine residues may also be involved in the structure formation with a three disulfide bridge and spätzle dimer. Generally, upon cleavage the Spätzle fragments form a dimer held together by intermolecular disulfide bridges (Weber et al., 2003).

To understand the functional role of TmSpzlb in Tenebrio innate immune responses against microbial challenge, three different experiments were designed. Although, the expression of $T m S p z 1 b$ mRNA was greater in adults than those in larval stages, we focused our experiments at the larval stage. We hypothesized that knocking down immune genes in the larvae was greater 
than in adults. Further, the larval stages in T. molitor have high industrial value such as food and feed. Under temporal distribution experiments, TmSpz1b was found to be induced more in hemocytes $6 \mathrm{~h}$ following the injection of $E$. coli, $S$. aureus, and C. albicans. In our previous studies we have used the same pathogens to identify the immunological role of Toll and IMD pathways through RNAi experiments (Keshavarz et al., 2020). In normal conditions, T. molitor challenged by these pathogens shows higher survival rate. Upon challenge by the same pathogens in a TmSpz1b dsRNA treated larvae, mortality increased. We hypothesized that these pathogens are effective in the functional characterization of T. molitor innate immune pathways. Spätzle proteins were initially expressed from the cells and localized in the hemocoel for a rapid response to produce AMPs. This has been studied in lepidopteran insects. In $M$. sexta, the spätzle gene was specifically induced by Grampositive bacterium $M$. luteus in hemocytes (An et al., 2010). Similarly, the A. pernyi spätzle (ApSpz) was induced by the Grampositive bacterium $E$. pernyi and the fungus, $N$. pernyi, but not by the Gram-negative bacterium E. coli (Sun et al., 2016). However, in B. mori, BmSpz1 was induced by E. coli, M. luteus, and fungi Saccharomyces cerevisiae (Wang et al., 2007). Furthermore, in the aquatic shrimp L. vannamei, LvSpz4 was induced by both $S$. aureus and $V$. alginolyticus (Yuan et al., 2017). In A. sinica, the spätzle gene belonging to the spätzle-4 family was induced by M. lysodeikticus (Zheng et al., 2012). Interestingly, PmSpz1 was induced by WSSV (Boonrawd et al., 2017). These results suggest that spätzle genes can be induced by different microorganisms. In the present study, the induction of TmSpz1b in response to $E$. coli infection may suggest a signaling cross-talk between the Toll and IMD pathways in T. molitor. T. molitor spätzle isoforms, such as TmSpz4 and TmSpz6, were temporally induced after E. coli infection, suggesting the role of AMPs in killing E. coli. Furthermore, based on this background, spätzle genes induced in hemocytes at $6 \mathrm{~h}$ post-injection of microorganisms may be involved in the secondary activation of the Toll pathway. It was reported that the spätzle produced from hemocytes, an immune organ, regulates production of AMPs from fat bodies in Drosophila (Lavine and Strand, 2002; Shia et al., 2009). Similarly, the hemocytes are the main organs that produce the TmSpzib protein in the mealworm.

Next, we characterized the effects of TmSpz1b RNAi on larval mortality against microbial challenge. E. coli, S. aureus, or C. albicans were injected into dsTmSpz1b-treated T. molitor larvae. Mortality of larvae injected with E. coli was significantly increased in dsTmSpz1b-treated T. molitor. Thus, TmSpz1b may have a critical immune function against $E$. coli infection. The results of the present study are consistent with our previous findings that TmSpz6 and TmSpz4 RNAi also increase larval mortality after E. coli challenge (Edosa et al., 2020a,b). Another valid observation is that knockdown of spätzle in the red palm weevil (Rhynchophorus ferrugineus) changes the composition of the gut bacteria, suggesting that spätzle might be involved in the homeostasis of the gut microbiota (Muhammad et al., 2020). We have not studied the knockdown of TmSpz1b in the gut of T. molitor and all our studies are valid for systemic infection in the whole larvae.
Finally, we explored the role of TmSpzlb in the innate immunity of T. molitor. The transcriptional regulation of 14 AMP genes were investigated in hemocytes, fat body, and gut tissues of TmSpz1b-silenced T. molitor larvae after $24 \mathrm{~h}$ exposure to E. coli, S. aureus, and C. albicans. Several of the AMP genes were positively regulated by $T m S p z 1 b$ in hemocytes and fat bodies in response to $E$. coli challenge, but not $S$. aureus and $C$. albicans challenges. TmTene1, TmAtt1a, TmAtt1b, TmColeA, TmColeB, and TmDef-like AMPs were positively regulated from hemocytes. TmAtt1a, TmAtt1b, TmColeA, TmColeB, and TmTLP2 AMPs were expressed in the fat bodies of T. molitor larvae after E. coli, but not $S$. aureus and $C$. albicans challenges in the survival assay. The downregulation of RfColeoptericin and RfDefensin was also confirmed in Rhynchophorus ferrugineus spätzle silenced larvae indicating that their secretion is under the regulation of the $R f$ Spätzle-mediated signaling pathway and was related to the compromising of $R$. ferrugineus innate immunity and maintenance of homeostasis of gut (Muhammad et al., 2020). This is interesting as Defensin secretion in Drosophila and Coleoptericin secretion in the cereal weevil Sitophilus are IMDdependent (Tingvall et al., 2001; Maire et al., 2018).

A previous study reported the altered expression of spätzle1A, a ligand for the Toll-like receptor, in Rel1-overexpressing or knockout mutants of $A$. aegypti. Furthermore, susceptibility to the entomopathogenic fungus $B$. bassiana was significantly increased in Rel1 knockout mutants (Bian et al., 2005). However, spätzle 5 in Drosophila acts as a ligand for the multi-ligand receptor Toll receptor 1 , and it is critical in antibacterial immunity against the Gram-positive bacterium Staphylococcus saprophyticus and the Gram-negative bacterium Erwinia carotovora carotovora 15 (Ecc15) (Nonaka et al., 2018). A co-immunoprecipitation assay indicated that the $M$. sexta Toll receptor (MsToll) can only bind to activated spätzle protein (C-108), and not inactive spätzle protein. Injection of recombinant C-108 induced several AMP genes, including drosomycin, cecropin, attacin, moricin, and lebocin, whereas injection of recombinant C-108 after treatment with MsTollspecific antibody could not activate AMP expression, suggesting that the Toll signaling pathway was activated by binding of the active form of spätzle to the Toll receptor (Zhong et al., 2012). In the present study co-immunoprecipitation or pulldown assays has not been conducted to prove the interaction between TmSpzlb and TmToll receptors. In shrimp, several studies sought to functionally characterize spätzle genes. In one study, FcSpz (F. chinensis spätzle) was induced by injection of both $V$. anguillarum and WSSV, and the AMP gene crustin 2 was upregulated by injection of recombinant FcSpz C-114 protein (the active form of $F c S p z$ protein) in crayfish (Shi et al., 2009). In addition, mortality against WSSV was significantly decreased by co-injection of recombinant PmSpzl protein, and the injection of recombinant $P m S p z l$ induced the expression of four AMP genes, including crustinPm1, crustinPm7, ALFPm3, and penaeidin3 (Boonrawd et al., 2017).

The studies on T. molitor Spätzle proteins have been fragmentary with individual studies on spätzle isoforms. During previous studies in T. molitor model, the extracellular Toll signaling pathway was fully characterized by biochemical studies 
with purified peptidoglycan from microorganisms (Kim et al., 2008; Roh et al., 2009; Yu et al., 2010). The polymeric diaminopimelic acid (DAP)-type peptidoglycan from Gramnegative bacteria can be recognized by the PGRP-SA complex, which activates spätzle in T. molitor (Yu et al., 2010; Keshavarz et al., 2020). In addition, TmCactin, the downstream component of Toll signaling pathway, plays an important role in innate immune responses against $E$. coli and $S$. aureus by positively regulating seven AMP genes (Jo et al., 2017). TmToll-7, one of the important Toll receptors, specifically regulates seven AMP genes to clear invading E. coli (Park et al., 2019). Prior studies have demonstrated that five AMP genes (TmTene1, TmDef-like, TmCole1, TmCole2, and TmAtt1b) are mainly regulated by Toll signaling-related genes (TmSpz1b, TmToll-7, and TmCactin) against E. coli challenge. These five AMP genes are mainly involved in sequestering of $E$. coli in the insect system (Table 2).

The collective findings indicate that TmSpzlb activated by E. coli positively regulates AMP genes in hemocytes and fat bodies. We propose an immune function of TmSpzlb in the mealworm. Thus, it is possible that TmSpzlb interacts with TmToll-7. This needs to be studied further using coimmunoprecipitation or pull-down assays. Further, we studied the expression of the NF-kB genes such as TmDorX2, and TmRelish in TmSpz-1b silenced individuals using qRT-PCR to establish the involvement of TmSpz-1b in T. molitor innate immunity related to the TLR-NF-kB pathway. The positive regulation of TmDor $X 2$ transcripts in hemocytes, fat body, and gut in TmSpz1b silenced individuals upon E. coli challenge substantiates the relationship between Spätzle and NF- $\mathrm{B}$ factor Dorsal within the Toll signaling pathway. We also propose that the upregulated AMP genes (negatively regulated) in TmSpz1bsilenced model-TmAtt2 and TmDef in hemocytes, TmTene4, TmAtt2, and TmDef-like in fat bodies, and TmTene1, TmTene2, TmTene4, TmAtt1b, TmAtt2, TmDef, TmDef-like, and TmCec2 in the gut-may be induced by another signaling pathway such as the IMD pathway, as well as a Toll signaling pathway induced by another Spätzle protein to maintain homeostasis. Interestingly, however, we have observed negative regulation of TmTene2 and TmAttala following silencing of TmCactin transcripts and E. coli challenge (Jo et al., 2017). In case of Tene2, it has been demonstrated that the production of this AMP is triggered by the Toll pathway through recognition of Gram-negative peptidoglycans (Roh et al., 2009; Yu et al., 2010), and an elevation of Tene 2 transcripts after TmCactin silencing could be attributed to the IMD signaling pathway. This leads to ask the most pertinent question- whether some AMPs are synergistically turned on by both Toll and

\section{REFERENCES}

Ali Mohammadie Kojour, M., Han, Y. S., and Jo, Y. H. (2020). An overview of insect innate immunity. Entomol. Res. 50, 282-291. doi: 10.1111/1748-5967. 12437

Ali Mohammadie Kojour, M., Jang, H. A., Edosa, T. T., Keshavarz, M., Kim, B. B., Bae, Y. M., et al. (2021). Identification, in silico characterization, and expression analysis of Tenebrio molitor Cecropin-2. Entomol. Res. 51, 74-82. doi: $10.1111 / 1748-5967.12476$
IMD pathways. Although the components of IMD pathway in T. molitor have been deciphered (Johnston et al., 2013), the IMD pathway is still elusive in this insect model. It would be interesting to note the effect of knockdown of IMD pathway components on IMD pathway and putative AMP gene expression. The information will contribute to the understanding of Toll and IMD pathway regulated AMP gene expression.

\section{CONCLUSION}

TmSpzlb is involved in the innate immunity of the mealworm beetle, T. molitor, by mediating the secretion of several AMPs in the beetle. These AMPs have a direct role in killing the Gramnegative bacterium $E$. coli in the hemocoel and reducing the mortality of T. molitor larvae.

\section{DATA AVAILABILITY STATEMENT}

Publicly available datasets were analyzed in this study. This data can be found here: GenBank/ XP015840683.1; XP_975083.1.

\section{AUTHOR CONTRIBUTIONS}

YJ and YH: conceptualization, methodology, visualization, and project administration. YH: software, validation, resources, data curation, supervision, and funding acquisition. $\mathrm{YB}$ and $\mathrm{YJ}$ : formal analysis. $\mathrm{YB}, \mathrm{BK}$, and $\mathrm{KP}$ : investigation. $\mathrm{YB}, \mathrm{TE}, \mathrm{MK}$, and MAK: writing-original draft preparation. BP, YL, and YH: writingreview and editing. All authors have read and agreed to the published version of the manuscript.

\section{FUNDING}

This research was supported by the Basic Science Research Program through the National Research Foundation of Korea (NRF) funded by the Ministry of Science, ICT and Future Planning (Grant No. 2018R1A2A2A05023367), and by Korea Institute of Planning and Evaluation for Technology in Food, Agriculture, Forestry and Fisheries (IPET) through Export Promotion Technology Development Program (Grant no. 617077-5), funded by the Ministry of Agriculture, Food and Rural Affairs (MAFRA).
An, C., Jiang, H., and Kanost, M. R. (2010). Proteolytic activation and function of the cytokine Spatzle in the innate immune response of a lepidopteran insect, Manduca sexta. FEBS J. 277, 148-162. doi: 10.1111/j.1742-4658.2009.07465.x

Anderson, K. V., Bokla, L., and Nusslein-Volhard, C. (1985). Establishment of dorsal-ventral polarity in the Drosophila embryo: the induction of polarity by the Toll gene product. Cell 42, 791-798. doi: 10.1016/0092-8674(85)90275-2

Arnot, C. J., Gay, N. J., and Gangloff, M. (2010). Molecular mechanism that induces activation of Spatzle, the ligand for the Drosophila Toll receptor. J. Biol. Chem. 285, 19502-19509. doi: 10.1074/jbc.M109.098186 
Bian, G., Shin, S. W., Cheon, H. M., Kokoza, V., and Raikhel, A. S. (2005). Transgenic alteration of Toll immune pathway in the female mosquito Aedes aegypti. Proc. Natl. Acad. Sci. U S A 102, 13568-13573. doi: 10.1073/pnas. 0502815102

Boonrawd, S., Mani, R., Ponprateep, S., Supungul, P., Masrinoul, P., Tassanakajon, A., et al. (2017). Characterization of PmSptzle 1 from the black tiger shrimp Peneaus monodon. Fish Shellfish Immunol. 65, 88-95. doi: 10.1016/j.fsi.2017. 04.005

Chae, J. H., Kurokawa, K., So, Y. I., Hwang, H. O., Kim, M. S., Park, J. W., et al. (2012). Purification and characterization of tenecin 4, a new anti-Gramnegative bacterial peptide, from the beetle Tenebrio molitor. Dev. Compar. Immunol. 36, 540-546. doi: 10.1016/j.dci.2011.09.010

Chasan, R., and Anderson, K. V. (1989). The role of easter, an apparent serine protease, in organizing the dorsal-ventral pattern of the Drosophila embryo. Cell 56, 391-400. doi: 10.1016/0092-8674(89)90242-0

De Gregorio, E., Spellman, P. T., Tzou, P., Rubin, G. M., and Lemaitre, B. (2002). The Toll and Imd pathways are the major regulators of the immune response in Drosophila. EMBO J. 21, 2568-2579. doi: 10.1093/emboj/21.11.2568

Edosa, T. T., Jo, Y. H., Keshavarz, M., Bae, Y. M., Kim, D. H., Lee, Y. S., et al. (2020a). TmSpz4 plays an important role in regulating the production of antimicrobial peptides in response to Escherichia coli and Candida albicans infections. Int. J. Mol. Sci. 21:1878. doi: 10.3390/ijms21051878

Edosa, T. T., Jo, Y. H., Keshavarz, M., Bae, Y. M., Kim, D. H., Lee, Y. S., et al. (2020b). TmSpz6 Is Essential for Regulating the Immune Response to Escherichia coli and Staphylococcus aureus Infection in Tenebrio molitor. Insects 11:105. doi: 10.3390/insects 11020105

Ferreira, A. G., Naylor, H., Esteves, S. S., Pais, I. S., Martins, N. E., and Teixeira, L. (2014). The Toll-Dorsal Pathway Is Required for Resistance to Viral Oral Infection in Drosophila. Plos Pathogens 10:e1004507. doi: 10.1371/journal.ppat. 1004507

Godfroy, J. I. III, Roostan, M., Moroz, Y. S., Korendovych, I. V., and Yin, H. (2012). Isolated Toll-like receptor transmembrane domains are capable of oligomerization. PLoS One 7:e48875. doi: 10.1371/journal.pone.0048875

Goel, M. K., Khanna, P., and Kishore, J. (2010). Understanding survival analysis: Kaplan-Meier estimate. Int. J. Ayurveda Res. 1, 274-278. doi: 10.4103/09747788.76794

Hoffmann, A., Funkner, A., Neumann, P., Juhnke, S., Walther, M., Schierhorn, A., et al. (2008a). Biophysical characterization of refolded Drosophila Spatzle, a cystine knot protein, reveals distinct properties of three isoforms. J. Biol. Chem. 283, 32598-32609. doi: 10.1074/jbc.M801815200

Hoffmann, A., Neumann, P., Schierhorn, A., and Stubbs, M. T. (2008b). Crystallization of Spatzle, a cystine-knot protein involved in embryonic development and innate immunity in Drosophila melanogaster. Acta Crystallogr. Sect. F Struct. Biol. Cryst. Commun. 64, 707-710. doi: 10.1107/ S1744309108018812

Hu, X., Yagi, Y., Tanji, T., Zhou, S., and Ip, Y. T. (2004). Multimerization and interaction of toll and spatzle in drosophila. Proc. Natl. Acad. Sci. U S A 101, 9369-9374. doi: 10.1073/pnas.0307062101

Imler, J. L., and Hoffmann, J. A. (2002). Toll receptors in Drosophila: a family of molecules regulating development and immunity. Curr. Top. Microbiol. Immunol. 270, 63-79. doi: 10.1007/978-3-642-59430-4_4

Issa, N., Guillaumot, N., Lauret, E., Matt, N., Schaeffer-Reiss, C., Van Dorsselaer, A., et al. (2018). The circulating protease persephone is an immune sensor for microbial proteolytic activities upstream of the drosophila toll pathway. Mol. Cell 69, 539-550. doi: 10.1016/j.molcel.2018.01.029

Jang, H. A., Park, K. B., Kim, B. B., Ali Mohammadie Kojour, M., Bae, Y. M., Baliarsingh, S., et al. (2020a). Bacterial but not fungal challenge up-regulates the transcription of Coleoptericin genes in Tenebrio molitor. Entomological Research 50, 440-449. doi: 10.1111/1748-5967.12465

Jang, H. A., Park, K. B., Kim, B. B., Ali Mohammadie Kojour, M., Bae, Y. M., Baliarsingh, S., et al. (2020b). In silico identification and expression analyses of Defensin genes in the mealworm beetle Tenebrio molitor. Entomol. Res. 50, 575-585. doi: 10.1111/1748-5967.12468

Jang, I. H., Chosa, N., Kim, S. H., Nam, H. J., Lemaitre, B., Ochiai, M., et al. (2006). A Spatzle-processing enzyme required for toll signaling activation in Drosophila innate immunity. Dev. Cell. 10, 45-55. doi: 10.1016/j.devcel.2005. 11.013
Jo, Y. H., Kim, Y. J., Park, K. B., Seong, J. H., Kim, S. G., Park, S., et al. (2017). TmCactin plays an important role in Gram-negative and -positive bacterial infection by regulating expression of 7 AMP genes in Tenebrio molitor. Sci. Rep. 7:46459. doi: 10.1038/srep46459

Jo, Y. H., Park, S., Park, K. B., Noh, M. Y., Cho, J. H., Ko, H. J., et al. (2018). In silico identification, characterization and expression analysis of attacin gene family in response to bacterial and fungal pathogens in Tenebrio molitor. Entomol. Res. 48, 45-54. doi: 10.1111/1748-5967.12287

Johnston, P. R., Makarova, O., and Rolff, J. (2013). Inducible defenses stay up late: temporal patterns of immune gene expression in Tenebrio molitor. G3 (Bethesda) 4, 947-955. doi: 10.1534/g3.113.008516

Jones, P., Binns, D., Chang, H. Y., Fraser, M., Li, W., McAnulla, C., et al. (2014). InterProScan 5: genome-scale protein function classification. Bioinformatics 30 , 1236-1240. doi: 10.1093/bioinformatics/btu031

Kawai, T., and Akira, S. (2010). The role of pattern-recognition receptors in innate immunity: update on Toll-like receptors. Nat. Immunol. 11, 373-384. doi: 10.1038/ni.1863

Keshavarz, M., Jo, Y. H., Edosa, T. T., Bae, Y. M., and Han, Y. S. (2020). TmPGRPSA regulates antimicrobial response to bacteria and fungi in the fat body and gut of Tenebrio molitor. Int. J. Mol. Sci. 21:2113. doi: 10.3390/ijms21062113

Kim, C. H., Kim, S. J., Kan, H., Kwon, H. M., Roh, K. B., Jiang, R., et al. (2008). A three-step proteolytic cascade mediates the activation of the peptidoglycaninduced toll pathway in an insect. J. Biol. Chem. 283, 7599-7607. doi: 10.1074/ jbc.M710216200

Kim, D. H., Lee, Y. T., Lee, Y. J., Chung, J. H., Lee, B. L., Choi, B. S., et al. (1998). Bacterial expression of tenecin 3, an insect antifungal protein isolated from Tenebrio molitor, and its efficient purification. Mol. Cells 8, 786-789.

Kim, D. H., Noh, M. Y., Park, K. B., and Jo, Y. H. (2017). Expression profiles of two thaumatin-like protein (TmTLP) genes in responses to various microorganisms from Tenebrio molitor. Entomol. Res. 47, 35-40. doi: 10.1111/17485967.12197

Kumar, S., Stecher, G., and Tamura, K. (2016). MEGA7: Molecular Evolutionary Genetics Analysis Version 7.0 for bigger datasets. Mol. Biol. Evol. 33, 1870-1874. doi: 10.1093/molbev/msw054

Larkin, M. A., Blackshields, G., Brown, N. P., Chenna, R., McGettigan, P. A., McWilliam, H., et al. (2007). Clustal W and Clustal X version 2.0. Bioinformatics 23, 2947-2948. doi: 10.1093/bioinformatics/btm404

Lavine, M., and Strand, M. (2002). Insect hemocytes and their role in immunity. Insect Biochem. Mol. Biol. 32, 1295-1309. doi: 10.1016/s0965-1748(02)00092-9

Lemaitre, B., Meister, M., Govind, S., Georgel, P., Steward, R., Reichhart, J. M., et al. (1995). Functional analysis and regulation of nuclear import of dorsal during the immune response in Drosophila. EMBO J. 14, 536-545. doi: 10.1002/j.14602075.1995.tb07029.x

Lemaitre, B., Nicolas, E., Michaut, L., Reichhart, J. M., and Hoffmann, J. A. (1996). The dorsoventral regulatory gene cassette spatzle/Toll/cactus controls the potent antifungal response in Drosophila adults. Cell 86, 973-983. doi: $10.1016 / \mathrm{s} 0092-8674(00) 80172-5$

Li, H., Li, T., Guo, Y., Li, Y., Zhang, Y., Teng, N., et al. (2018). Molecular characterization and expression patterns of a non-mammalian toll-like receptor gene (TLR21) in larvae ontogeny of common carp (Cyprinus carpio L.) and upon immune stimulation. BMC Vet. Res. 14:153. doi: 10.1186/s12917-018$1474-4$

Livak, K. J., and Schmittgen, T. D. (2001). Analysis of relative gene expression data using real-time quantitative PCR and the 2(T)(-Delta Delta C) method. Methods 25, 402-408. doi: 10.1006/meth.2001.1262

Maire, J., Vincent-Monegat, C., Masson, F., Zaidman-Remy, A., and Heddi, A. (2018). An IMD-like pathway mediates both endosymbiont control and host immunity in the cereal weevil Sitophilus spp. Microbiome 6:6. doi: 10.1186/ s40168-017-0397-9

Medzhitov, R. (2001). Toll-like receptors and innate immunity. Nat. Rev. Immunol. 1, 135-145. doi: 10.1038/35100529

Michel, T., Reichhart, J. M., Hoffmann, J. A., and Royet, J. (2001). Drosophila Toll is activated by Gram-positive bacteria through a circulating peptidoglycan recognition protein. Nature 414, 756-759. doi: 10.1038/414756a

Morisato, D. (2001). Spatzle regulates the shape of the Dorsal gradient in the Drosophila embryo. Development 128, 2309-2319. doi: 10.1242/dev.128.12. 2309 
Mount, D. W. (2007). Using the Basic Local Alignment Search Tool (BLAST). CSH Protoc. 2007 2007:db.to17. doi: 10.1101/pdb.top17

Muhammad, A., Habineza, P., Wang, X., Xiao, R., Ji, T., Hou, Y., et al. (2020). Spatzle homolog-mediated toll-like pathway regulates innate immune responses to maintain the homeostasis of gut microbiota in the red palm weevil, Rhynchophorus ferrugineus Olivier (Coleoptera: Dryophthoridae). Front. Microbiol. 11:846. doi: 10.3389/fmicb.2020.00846

Mulinari, S., Hacker, U., and Castillejo-Lopez, C. (2006). Expression and regulation of Spatzle-processing enzyme in Drosophila. FEBS Lett. 580, 5406-5410. doi: 10.1016/j.febslet.2006.09.009

Nakamoto, M., Moy, R. H., Xu, J., Bambina, S., Yasunaga, A., Shelly, S. S., et al. (2012). Virus recognition by Toll-7 activates antiviral autophagy in Drosophila. Immunity 36, 658-667. doi: 10.1016/j.immuni.2012.03.003

Nie, L., Cai, S. Y., Shao, J. Z., and Chen, J. (2018). Toll-like receptors, associated biological roles, and signaling networks in non-mammals. Front. Immunol. 9:1523. doi: 10.3389/fimmu.2018.01523

Noh, M. Y., and Jo, Y. H. (2016). Identification and sequence analysis of two thaumatin-like protein (TmTLP) genes from Tenebrio molitor. Entomol. Res. 46, 354-359. doi: 10.1111/1748-5967.12198

Nonaka, S., Kawamura, K., Hori, A., Salim, E., Fukushima, K., Nakanishi, Y., et al. (2018). Characterization of Spz5 as a novel ligand for Drosophila Toll-1 receptor. Biochem. Biophys. Res. Commun. 506, 510-515. doi: 10.1016/j.bbrc. 2018.10.096

Park, S., Jo, Y. H., Park, K. B., Ko, H. J., Kim, C. E., Bae, Y. M., et al. (2019). TmToll7 plays a crucial role in innate immune responses against gram-negative bacteria by regulating 5 AMP genes in Tenebrio molitor. Front. Immunol. 10:310. doi: 10.3389/fimmu.2019.00310

Parker, J. S., Mizuguchi, K., and Gay, N. J. (2001). A family of proteins related to Spatzle, the toll receptor ligand, are encoded in the Drosophila genome. Proteins 45, 71-80. doi: 10.1002/prot.1125

Roh, K. B., Kim, C. H., Lee, H., Kwon, H. M., Park, J. W., Ryu, J. H., et al. (2009). Proteolytic cascade for the activation of the insect toll pathway induced by the fungal cell wall component. J. Biol. Chem. 284, 19474-19481. doi: 10.1074/jbc. M109.007419

Schneider, D. S., Jin, Y., Morisato, D., and Anderson, K. V. (1994). A processed form of the Spatzle protein defines dorsal-ventral polarity in the Drosophila embryo. Development 120, 1243-1250. doi: 10.1242/dev.120.5.1243

Shaukat, Z., Liu, D., and Gregory, S. (2015). Sterile inflammation in Drosophila. Mediat. Inflamm. 2015:369286. doi: 10.1155/2015/369286

Shi, X. Z., Zhang, R. R., Jia, Y. P., Zhao, X. F., Yu, X. Q., and Wang, J. X. (2009). Identification and molecular characterization of a Spatzle-like protein from Chinese shrimp (Fenneropenaeus chinensis). Fish Shellfish Immunol. 27, 610-617. doi: 10.1016/j.fsi.2009.07.005

Shia, A. K., Glittenberg, M., Thompson, G., Weber, A. N., Reichhart, J. M., and Ligoxygakis, P. (2009). Toll-dependent antimicrobial responses in Drosophila larval fat body require Spatzle secreted by haemocytes. J. Cell. Sci. 122, 45054515. doi: $10.1242 /$ jcs. 049155

Shin, S. W., Bian, G., and Raikhel, A. S. (2006). A toll receptor and a cytokine, Toll5A and Spz1C, are involved in toll antifungal immune signaling in the mosquito Aedes aegypti. J. Biol. Chem. 281, 39388-39395. doi: 10.1074/jbc. M608912200

Sun, Y., Jiang, Y., Wang, Y., Li, X., Yang, R., Yu, Z., et al. (2016). The toll signaling pathway in the Chinese oak silkworm, antheraea pernyi: innate immune responses to different microorganisms. PLoS One 11:e0160200. doi: 10.1371/journal.pone. 0160200

Tingvall, T. O., Roos, E., and Engstrom, Y. (2001). The imd gene is required for local Cecropin expression in Drosophila barrier epithelia. Embo Rep. 2, 239-243. doi: 10.1093/embo-reports/kve048

Vaniksampanna, A., Longyant, S., Charoensapsri, W., Sithigorngul, P., and Chaivisuthangkura, P. (2019). Molecular isolation and characterization of a spatzle gene from Macrobrachium rosenbergii. Fish Shellfish Immunol. 84, 441-450. doi: 10.1016/j.fsi.2018.10.015

Wang, Y., Cheng, T. C., Rayaprolu, S., Zou, Z., Xia, Q. Y., Xiang, Z. H., et al. (2007). Proteolytic activation of pro-spatzle is required for the induced transcription of antimicrobial peptide genes in lepidopteran insects. Dev. Compar. Immunol. 31, 1002-1012. doi: 10.1016/j.dci.2007.01.001

Weber, A. N. R., Tauszig-Delamasure, S., Hoffmann, J. A., Lelievre, E., Gascan, H., Ray, K. P., et al. (2003). Binding of the Drosophila cytokine Spatzle to Toll is direct and establishes signaling. Nat. Immunol. 4, 794-800. doi: 10.1038/ ni955

Yamamoto-Hino, M., and Goto, S. (2016). Spatzle-Processing Enzymeindependent Activation of the Toll Pathway in Drosophila Innate Immunity. Cell. Struct. Funct. 41, 55-60. doi: 10.1247/csf.16002

Yang, Y. T., Lee, M. R., Lee, S. J., Kim, S., Nai, Y. S., and Kim, J. S. (2017). Tenebrio molitor Gram-negative-binding protein 3 (TmGNBP3) is essential for inducing downstream antifungal Tenecin 1 gene expression against infection with Beauveria bassiana JEF-007. Insect Sci. 25, 969-977. doi: 10.1111/17447917.12482

Yu, D., Wu, Y., Xu, L., Fan, Y., Peng, L., Xu, M., et al. (2016). Identification and characterization of toll-like receptors (TLRs) in the Chinese tree shrew (Tupaia belangeri chinensis). Dev. Comp. Immunol. 60, 127-138. doi: 10.1016/j.dci. 2016.02.025

Yu, M., Zhang, Y., Tang, X., Ren, J., and Zhang, Y. (2015). The first mollusk spatzle homolog gene in the clam, Paphia undulate. Fish Shellfish Immunol. 47, 712-716. doi: 10.1016/j.fsi.2015.10.017

Yu, Y., Park, J. W., Kwon, H. M., Hwang, H. O., Jang, I. H., Masuda, A., et al. (2010). Diversity of innate immune recognition mechanism for bacterial polymeric meso-diaminopimelic acid-type peptidoglycan in insects. J. Biol. Chem. 285, 32937-32945. doi: 10.1074/jbc.M110.144014

Yuan, K., Yuan, F. H., Weng, S. P., He, J. G., and Chen, Y. H. (2017). Identification and functional characterization of a novel Spatzle gene in Litopenaeus vannamei. Dev. Comp. Immunol. 68, 46-57. doi: 10.1016/j.dci.2016.11.016

Zheng, L. P., Hou, L., Yu, M., Li, X., and Zou, X. Y. (2012). Cloning and the expression pattern of Spatzle gene during embryonic development and bacterial challenge in Artemia sinica. Mol. Biol. Rep. 39, 6035-6042. doi: 10.1007/ s11033-011-1417-7

Zhong, X., Xu, X. X., Yi, H. Y., Lin, C., and Yu, X. Q. (2012). A Toll-Spatzle pathway in the tobacco hornworm, Manduca sexta. Insect Biochem. Mol. Biol. 42, 514-524. doi: 10.1016/j.ibmb.2012.03.009

Zhu, J. Y., Wu, G. X., and Zhang, Z. (2014). Upregulation of coleoptericin transcription in Tenebrio molitor parasitized by Scleroderma guani. J. AsiaPacific Entomol. 17, 339-342. doi: 10.1016/j.aspen.2014.03.001

Conflict of Interest: The authors declare that the research was conducted in the absence of any commercial or financial relationships that could be construed as a potential conflict of interest.

Publisher's Note: All claims expressed in this article are solely those of the authors and do not necessarily represent those of their affiliated organizations, or those of the publisher, the editors and the reviewers. Any product that may be evaluated in this article, or claim that may be made by its manufacturer, is not guaranteed or endorsed by the publisher.

Copyright (c) 2021 Bae, Jo, Patnaik, Kim, Park, Edosa, Keshavarz, Kojour, Lee and Han. This is an open-access article distributed under the terms of the Creative Commons Attribution License (CC BY). The use, distribution or reproduction in other forums is permitted, provided the original author(s) and the copyright owner(s) are credited and that the original publication in this journal is cited, in accordance with accepted academic practice. No use, distribution or reproduction is permitted which does not comply with these terms. 